\title{
First-principles study of point defects in an fcc Fe-10Ni-20Cr model alloy
}

\author{
J. B. Piochaud, ${ }^{1,2}$ T. P. C. Klaver, ${ }^{3}$ G. Adjanor, ${ }^{2,4}$ P. Olsson, ${ }^{5}$ C. Domain, ${ }^{2,4}$ and C. S. Becquart ${ }^{1,2}$ \\ ${ }^{1}$ Université des Sciences et Technologies de Lille, Bâtiment C6, F-59655 Villeneuve d'Ascq Cedex, France \\ ${ }^{2}$ Laboratoire commun EDF-CNRS Etude et Modélisation des Microstructures pour le Vieillissement des Matériaux (EM2VM), France \\ ${ }^{3}$ Department of Materials Science and Engineering, Delft University of Technology, Mekelweg 2, 2628 CD Delft, The Netherlands \\ ${ }^{4}$ Electricité de France, EDF Recherche et Développement, Département Matériaux et Mécanique des Composants, \\ Les Renardières, F-77250 Moret sur Loing, France \\ ${ }^{5}$ KTH Royal Institute of Technology, Reactor Physics, Roslagstullsbacken 21, SE-10691 Stockholm, Sweden
}

(Received 20 June 2013; published 3 January 2014)

\begin{abstract}
The influence of the local environment on vacancy and self-interstitial formation energies has been investigated in a face-centered-cubic (fcc) $\mathrm{Fe}-10 \mathrm{Ni}-20 \mathrm{Cr}$ model alloy by analyzing an extensive set of first-principle calculations based on density functional theory. Chemical disorder has been considered by designing special quasirandom structures and four different collinear magnetic structures have been investigated in order to determine a relevant reference state to perform point defect calculations at $0 \mathrm{~K}$. Two different convergence methods have also been used to characterize the importance of the method on the results. Although our fcc Fe-10Ni-20Cr would be better represented in terms of applications by the paramagnetic state, we found that the antiferromagnetic single-layer magnetic structure was the most stable at $0 \mathrm{~K}$ and we chose it as a reference state to determine the point defect properties. Point defects have been introduced in this reference state, i.e., vacancies and $\mathrm{Fe}-\mathrm{Fe}, \mathrm{Fe}-\mathrm{Ni}, \mathrm{Fe}-\mathrm{Cr}, \mathrm{Cr}-\mathrm{Cr}$, Ni-Ni, and $\mathrm{Ni}-\mathrm{Cr}$ dumbbell interstitials oriented either parallel or perpendicular to the single layer antiferromagnetic planes. Each point defect studied was introduced at different lattice sites to consider a sufficient variety of local environments and analyze its influence on the formation energy values. We have estimated the point defect formation energies with linear regressions using variables which describe the local environment surrounding the point defects. The number and the position of $\mathrm{Ni}$ and $\mathrm{Cr}$ first nearest neighbors to the point defects were found to drive the evolution of the formation energies. In particular, $\mathrm{Ni}$ is found to decrease and $\mathrm{Cr}$ to increase the vacancy formation energy of the model alloy, while the opposite trends are found for the dumbbell interstitials. This study suggested that, to a first approximation, the first nearest atoms to point defects can provide reliable estimates of point defect formation energies.
\end{abstract}

DOI: 10.1103/PhysRevB.89.024101

PACS number(s): 61.82.Bg, 71.15.Mb, 75.50.Bb

\section{INTRODUCTION}

Stainless steels (SS) are key materials in a wide range of applications. ${ }^{1}$ These steels contain at least 11 wt $\%$ of chromium $(\mathrm{Cr})$ responsible for their stainless properties. Austenitic SS are widely used in the nuclear industry because of their good mechanical properties and their superior corrosion resistance over a wide range of temperature. In particular, austenitic SS of types 304 and 316 are used, among other things, as a primary containment barrier protecting the reactor pressure vessels from irradiation damage. These internal structures are quenched in a metastable phase which contains a high proportion of austenitic solid solution. These Fe-based alloys contain a maximum of 0.15 wt $\%$ carbon, a minimum of 16 wt \% chromium and sufficient nickel (about $10 \mathrm{wt} \%$ ) to retain a face-centered-cubic (fcc) crystallographic structure from the cryogenic temperature region to the melting point of the alloy. Many solute atoms are also included (Mn, Si, $\mathrm{P}, \mathrm{S}, \mathrm{Mo}$, and $\mathrm{N}$ ) to improve their performance under specific operating conditions. Finally, these steels are paramagnetic (PM) at the operating temperatures of nuclear power reactors.

In service, internal structures are exposed to harsh conditions: temperatures around $350{ }^{\circ} \mathrm{C}$, neutron irradiation, mechanical and thermal stresses, and a corrosive environment. This combination of factors may allow, among other effects, the occurrence of creep, swelling, and radiation induced segregation, thus diminishing the performance of the internals with time. The combination of all these phenomena may lead to the occurrence of irradiation assisted stress corrosion which may initiate cracks in the baffle bolts that assist in maintaining the structural integrity of the core. It is now well understood that the above-mentioned macroscopic changes depend on the impact of irradiation on the defect production in these materials. In particular, it depends on how and in what proportion the point defects (vacancies and interstitials) can cluster into larger defects (dislocations, loops, voids, etc.) and affect the microstructural evolution of the material. Thus, the structure and mobility of the point defects as well as their interactions with the atoms of the major or minor elements must be addressed to provide insight into the mechanisms involved under irradiation.

However, experimental data on point defects are scarce in austenitic SS (Refs. 2-6) owing to the difficulty in precisely determining atomic quantities ${ }^{7}$ such as the formation and migration energies of a single vacancy or that of an interstitial. These physical properties can also be greatly affected by impurities $^{5}$ and the influence of the local environment is difficult to properly probe.

In this context, density functional theory (DFT) may help to better interpret and understand experimental results. It could seem simpler to determine these quantities theoretically, however in the case of austenitic steels, the task of choosing a relevant reference state is not trivial. In particular, the ground state of Fe is body centered cubic (bcc) whereas the austenitic steels, whose major element is Fe, are fcc at operating conditions. Thus, it is difficult to conduct experimental studies 
of defect and solute properties in dilute fcc Fe. Considerable experimental efforts have therefore been carried out to stabilize the $\gamma$-Fe at lower temperature ${ }^{8-10}$ ( $\mathrm{Fe}$ is fcc at $\left.1185-1664 \mathrm{~K}\right)$ to improve the current level of understanding by studying the magnetic ordering. These experiments have shown that $\gamma-\mathrm{Fe}$ displays a noncollinear, spiral magnetic structure. ${ }^{11,12}$

The numerous first-principles studies carried out to search for the $\gamma$-Fe ground state ${ }^{13-20}$ showed many competing magnetic structures lying between 0.08 and $0.15 \mathrm{eV} /$ atom above the bcc ferromagnetic ground state, thus complicating the choice of a relevant reference state for calculations of physical properties in $\gamma$-Fe. Another critical issue is to model the PM state in $\gamma$-Fe since austenitic SS are PM at temperatures where nuclear reactors operate. However, a difficulty arises at $0 \mathrm{~K}$ where austenitic SS seem to be antiferromagnetic (AFM) ${ }^{21}$ which is certainly not the most relevant state in order to be representative of a high temperature PM state. Furthermore, reliable first-principles calculations of the PM state are not trivial ${ }^{22}$ and there remains some debate about whether the paramagnetism is better represented as itinerant or involves localized moments on the ions. In this context, a relevant strategy used by Klaver et al. ${ }^{23}$ in dilute austenitic $\mathrm{Fe}-\mathrm{Ni}-\mathrm{Cr}$ alloys was to calculate some physical properties in multiple collinear magnetic structures selected as possible reference states for austenite, and then retain features common among these states in order to extrapolate them to the PM state.

Finally, it is also important to consider the multicomponent nature of austenitic $\mathrm{Fe}-\mathrm{Ni}-\mathrm{Cr}$ alloys. $\mathrm{Cr}$ has a bcc crystal structure and exhibits an incommensurate spin-density wave at low temperatures, which is not trivial to properly capture in DFT. ${ }^{24-26} \mathrm{Ni}$ is fcc and displays a ferromagnetic (FM) ordering at low temperatures. This large difference between the properties of the pure elements is responsible for the wide variety of magnetic phases in the ternary phase diagram. In particular, at low temperatures, the magnetic phase of $\gamma-\mathrm{Fe}_{80-x} \mathrm{Ni}_{x} \mathrm{Cr}_{20}$ alloys $(10<x<30)$ can have an antiferromagnetic phase, a spin-glass ordering, or a mixture between spin-glass ordering and FM ordering as the Ni content increases. $^{21}$

Despite the difficulties presented above, first-principles calculations still have a key role to play in such a context due to their ability to provide predictive information about the complex interplay occurring between the electronic and structural degrees of freedom in complex systems. To our knowledge, DFT studies of concentrated austenitic Fe-Ni-Cr alloys are few ${ }^{27-31}$ and are only based on the coherent potential approximation (CPA). In this work, we model the austenitic steels by designing special quasirandom structures (SQS) ${ }^{32}$ formed by the major alloying elements of 304 and 316 grades with the same average composition, i.e., respectively, 70 at. $\% \mathrm{Fe}, 20$ at. $\% \mathrm{Cr}$, and 10 at. \% Ni. This simplified ternary system still remains very complex because its elements display very different properties, as shown previously. However, from a fundamental point of view austenitic $\mathrm{Fe}-\mathrm{Cr}-\mathrm{Ni}$ alloys are key model systems that can be used in order to investigate the complex magnetostructural behavior of austenitic steels. In particular, the chemical disorders as well as the magnetic properties need to be considered to explain a wide range of properties of these alloys. We thus investigated in this work a wide set of DFT calculations performed in a $\mathrm{Fe}-10 \mathrm{Ni}-20 \mathrm{Cr}$ alloy used as model austenitic steel. In particular, we analyze the influence of the local environments on both vacancy and $\langle 100\rangle$ dumbbell formation energies in fcc metals and dilute Fe-Cr-Ni alloys. ${ }^{23,33-37}$

The paper is organized as follows. Section II contains the computational details used to perform the DFT calculations. In particular, attention is paid to the calculation of point defect formation energies in concentrated alloys. In Sec. III we present and discuss the stability of four different magnetic phases in order to calculate the point defect formation energies in the Fe-10Ni-20Cr model alloy. In Sec. IV, the link found between the local environment and the point defect formation energies calculated in the selected reference state is presented. Finally, we present our conclusions in the last section.

\section{COMPUTATIONAL DETAILS}

The first-principles calculations were performed using the DFT code VASP, ${ }^{38,39}$ a plane-wave code that implements the projector augmented wave (PAW) method. ${ }^{40,41}$ The standard PAW potentials supplied with VASP were used. Exchange and correlation were described by the Perdew-Wang functional, ${ }^{42}$ adding a nonlocal correction in the form of the generalized gradient approximation. The Vosko-Wilk-Nusair interpolation was applied for the correlation part of the exchange-correlation functional. Potentials with eight, six, and ten valence electrons were used for $\mathrm{Fe}, \mathrm{Cr}$, and $\mathrm{Ni}$, respectively. The blocked Davidson algorithm ${ }^{43}$ was used to minimize the energy of the system.

Two sets of calculations were performed in this work. Reference state calculations were performed in supercells of 256 atoms containing $180 \mathrm{Fe}$ atoms (70 at. \%), $50 \mathrm{Cr}$ atoms (20 at. $\%$ ), and $26 \mathrm{Ni}$ atoms (10 at. \%). In this first set of calculations, the local magnetic moments on atoms were initialized to impose the desired magnetic ordering and then allowed to relax. Four different collinear magnetic structures were compared to select a relevant reference state for the point defect calculations at $0 \mathrm{~K}$. As the PM state is certainly the most relevant state to describe the high temperature state, it was modeled by generating two different SQSs (SQSa and SQSb) for the six-component $\left(\mathrm{Fe}_{0.5}^{\uparrow} \mathrm{Fe}_{0.5}^{\downarrow}\right)_{70}\left(\mathrm{Ni}_{0.5}^{\uparrow} \mathrm{Ni}_{0.5}^{\downarrow}\right)_{10}\left(\mathrm{Cr}_{0.5}^{\uparrow} \mathrm{Cr}_{0.5}^{\downarrow}\right)_{20}$ alloy to take into account both chemical and magnetic disorder in the lattice. As has been shown in $\mathrm{Cr}-\mathrm{N},{ }^{44}$ the use of SQSs to treat magnetic disordering is well justified from a practical point of view. However, the choice of applying the adiabatic approximation also to the magnetically disordered structure relaxations should overestimate the formation energy of the structure. ${ }^{45}$ As will be further outlined, the defect calculations are performed in the AFM1 structure so that this issue does not affect the results or conclusions.

From these SQSs, three other magnetic states were built by initializing the moments to impose the desired magnetic ordering. As the AFM state may be the ground magnetic state of the fcc $\mathrm{Fe}-10 \mathrm{Ni}-20 \mathrm{Cr}$ alloy, we considered the single-layer antiferromagnetic (AFM1) state in which (100) planes contain moments in directions alternatingly parallel and antiparallel ( $\downarrow \uparrow \downarrow \ldots)$, and the double-layer antiferromagnetic (AFMD) state in which pairs of (100) planes contain moments in directions alternatingly parallel and antiparallel $(\uparrow \uparrow \downarrow \downarrow \ldots)$. 
Finally, for comparison purposes, we also considered the FM state in which all moments point in the same direction. In a second set of calculations, formation energies of point defects were calculated in supercells of $256 \pm 1$ atoms, with supercell dimensions held fixed at the equilibrium values of the reference state selected and ionic positions free to relax.

For the reference state calculations, two different calculation methods were considered in order to analyze its impact on the observed properties. Fully relaxed (FR) calculations were performed in which the atomic coordinates and the supercell shape and volume were allowed to relax. Cubic lattice (CL) calculations were also performed in which the lattice maintained fcc symmetry and only the atomic coordinates and the supercell volume were allowed to relax.

The local magnetic moments were determined by integrating the spin density within spheres centered on the atoms using sphere radii of $1.302,1.323$, and $1.286 \AA$ for $\mathrm{Fe}, \mathrm{Cr}$, and $\mathrm{Ni}$, respectively.

All the above-mentioned sets of calculations were performed using an energy cutoff of $300 \mathrm{eV}$ for the plane-wave basis set and a $2 \times 2 \times 2$ Monkhorst-Pack grid to sample the Brillouin zone. First-order Methfessel and Paxton smearing ${ }^{46}$ of the Fermi surface was used throughout with a smearing width set to $0.3 \mathrm{eV}$.

Vacancies and $\langle 100\rangle$ dumbbells were considered for the point defect calculations. These calculations were performed in the AFM1 state which was selected as the reference state as we will show next. Vacancies have been introduced by removing one $\mathrm{Fe}$ atom from the reference state and dumbbells have been introduced by replacing one $\mathrm{Fe}$ atom from the reference state by two atoms. Point defect calculations were performed at many sites (at least 40 sites) to analyze the impact of the local environment on formation energies. For comparison purposes, vacancies and interstitials were introduced on the same sites of the AFM1 reference state. All the possible configurations for the dumbbell were considered, i.e., Fe-Fe, Fe-Ni, Fe-Cr, $\mathrm{Cr}-\mathrm{Cr}$, Ni-Ni, and $\mathrm{Ni}-\mathrm{Cr}$ dumbbells oriented either parallel (oriented along the [010] axis) or perpendicular (oriented along the [100] axis) to the AFM1 magnetic planes. The point defect formation energies were calculated by calculating the difference between the energy of the defect lattice (either $E_{N-1}$ for the vacancies or $E_{N+1}$ for the dumbbells), and the energy of the reference state $E_{N}$, where $N$ refers to the number of atoms of the reference state (256 atoms). However, as these lattices do not have the same number of atoms, we need to define the chemical potentials for one $\mathrm{Fe}$ atom $\mu_{\mathrm{Fe}}$, one $\mathrm{Ni}$ atom $\mu_{\mathrm{Ni}}$, and one $\mathrm{Cr}$ atom $\mu_{\mathrm{Cr}}$ to be able to calculate all the point defect energies as follows:

$$
\begin{gathered}
E_{f}^{V}=E_{N-1}-\left(E_{N}-\mu_{\mathrm{Fe}}\right), \\
E_{f}^{\mathrm{Fe}-A}=E_{N+1}-\left(E_{N}+\mu_{A}\right), \\
E_{f}^{A-B}=E_{N+1}-\left(E_{N}-\mu_{\mathrm{Fe}}+\mu_{A}+\mu_{B}\right),
\end{gathered}
$$

where $E_{f}^{V}$ is the formation energy of a vacancy, $E_{f}^{\mathrm{Fe}-A}$ is the formation energy of a mixed Fe- $A$ dumbbell, and $E_{f}^{A-B}$ is the formation energy of an $A-B$ dumbbell ( $A$ and $B$ refer to elements different than $\mathrm{Fe}$ ). Indeed, in a concentrated $\mathrm{Fe}-\mathrm{Ni}-\mathrm{Cr}$ system, we can expect the chemical potentials defined above to be substantially different from the chemical potentials of elements in their pure reference states. Thus, for the purpose of calculating defect formation energies from Eq. (1) to Eq. (3), calculations of the chemical potentials of the species involved in the defects are required. At finite temperature, the excess chemical potential differences are usually calculated using Widom-type substitution techniques. ${ }^{47}$ This consists of simulating the nonsubstituted reference system " $A$ " of energy $U_{A}$ (containing $N_{A}$ atoms of type $A$ and $N_{B}$ atoms of type $B$ ) with a Monte Carlo procedure, and estimating the excess chemical potential difference by canonical averages of the exponential of the substitution energy $\Delta^{A \rightarrow B} E=$ $E_{B}-E_{A}$. Precise calculations of chemical potentials by DFT methods would be extremely time consuming. This would require, depending on temperature, sampling a large number of configurations by a Monte Carlo Metropolis procedure. In Appendix, we suggest an approach to calculate these quantities while feasible in terms of calculation time. When applying this method to estimate the reference chemical potential of $\mathrm{Fe}, \mathrm{Ni}$, and $\mathrm{Cr}$ in the AFM1 Fe-10Ni-20Cr reference state, we obtain the three differences in reference chemical potentials as follows:

$$
\begin{aligned}
& \mu_{\mathrm{Cr}}^{\mathrm{Fe}-10 \mathrm{Ni}-20 \mathrm{Cr}}-\mu_{\mathrm{Fe}}^{\mathrm{Fe}-10 \mathrm{Ni}-20 \mathrm{Cr}}=\frac{1}{2}\left(\Delta^{\mathrm{Fe} \rightarrow \mathrm{Cr}} \mu-\Delta^{\mathrm{Cr} \rightarrow \mathrm{Fe}} \mu\right), \\
& \mu_{\mathrm{Ni}}^{\mathrm{Fe}-10 \mathrm{Ni}-20 \mathrm{Cr}}-\mu_{\mathrm{Fe}}^{\mathrm{Fe}-10 \mathrm{Ni}-20 \mathrm{Cr}}=\frac{1}{2}\left(\Delta^{\mathrm{Fe} \rightarrow \mathrm{Ni}} \mu-\Delta^{\mathrm{Ni} \rightarrow \mathrm{Fe}} \mu\right), \\
& \mu_{\mathrm{Ni}}^{\mathrm{Fe}-10 \mathrm{Ni}-20 \mathrm{Cr}}-\mu_{\mathrm{Cr}}^{\mathrm{Fe}-10 \mathrm{Ni}-20 \mathrm{Cr}}=\frac{1}{2}\left(\Delta^{\mathrm{Cr} \rightarrow \mathrm{Ni}} \mu-\Delta^{\mathrm{Ni} \rightarrow \mathrm{Cr}} \mu\right),
\end{aligned}
$$

where $\Delta^{A \rightarrow B} \mu$ is the minimum of the " $A$ to $B$ " substitution energies and $\mu_{A}^{\mathrm{Fe}-10 \mathrm{Ni}-20 \mathrm{Cr}}$ is the estimate of chemical potentials in VASP for the addition or the removal of atom $A$ in our AFM1 reference state. We thus calculated the minimum of the substitution energy on most of the $180 \mathrm{Fe}, 50 \mathrm{Cr}$, and $26 \mathrm{Ni}$ atom sites. Advanced estimation techniques which rigorously combine so-called "forward" and "backward" estimates $\Delta^{A \rightarrow B} \mu$ and $-\Delta^{B \rightarrow A} \mu$ (Refs. 48 and 49) cannot be applied in the present method, as no actual Monte Carlo sampling is done in this simplified approach, and a simple arithmetic average is taken in the previous equations. This system of three equations [from Eq. (4) to Eq. (6)] provides in fact only two independent equations, the last one being the difference of the former two. It cannot be used to obtain the values of the chemical potentials, but only to check the reliability of the estimates (if the numerical estimate yielded by the difference of the first two is close to the estimate of the last one, this supports the accuracy of the estimates, as they were not calculated with the same substitutions). The total energy of the system calculated with VASP provides the additional equation needed to estimate the reference chemical potentials [Eq. (7)]. Indeed, as the calculations were made at constant volume, and neglecting all entropic contributions, we have $E_{A} \approx F(V)$ and

$$
F(V)=\sum_{A} N_{A} \mu_{A}^{\mathrm{Fe}-10 \mathrm{Ni}-20 \mathrm{Cr}} .
$$




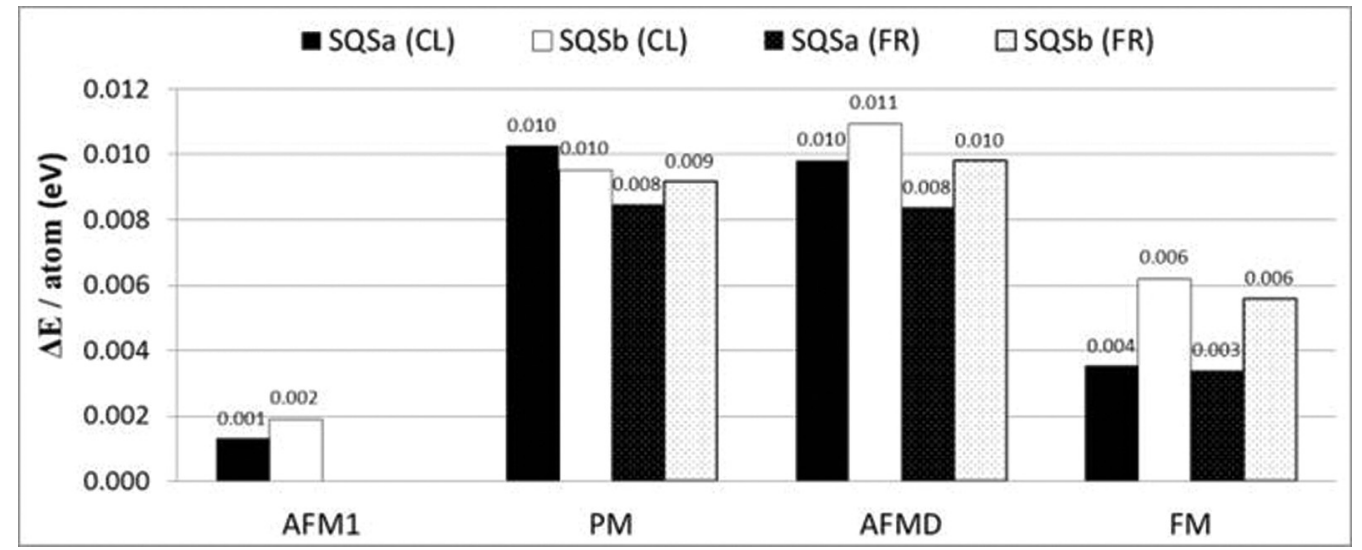

FIG. 1. For each SQS, the energy per atom, $\Delta E$, for the distinct magnetic states studied, is given relative to the AFM1 state calculated using the FR calculation method.

\section{REFERENCE STATE CALCULATIONS}

Different spin configurations were investigated using DFT. In particular, tests were performed with unconstrained spin ordering and it was seen that configurations that were initially noncollinear, converged to collinear states. We thus present only results obtained with collinear spin configurations in this work. As mentioned previously, we initialized four different magnetic states which have been tested in two different SQSs (SQSa and SQSb). As already found in previous works for $\gamma-\mathrm{Fe},{ }^{13-17}$ we found many minima very close in energy corresponding to different magnetic states as can be seen in Fig. 1. The energy differences of a few meV per atom observed between FR and CL methods, are due to the fact that both ferromagnetic and antiferromagnetic states transform into face-centered-tetragonal (fct) structures when the CL constraint is removed. By contrast, the crystallographic phase remains fcc for the PM state. The AFM1 state is found to have the lowest energy, in agreement with the detailed magnetic measurements performed in polycrystalline $\mathrm{Fe}_{80-x} \mathrm{Ni}_{x} \mathrm{Cr}_{20}$ alloys by Majumdar et al. at the composition of the Fe-10Ni$20 \mathrm{Cr}$ model alloy at $0 \mathrm{~K} .^{21}$

One interesting result obtained from these calculations is that we found that the relaxed moments carried by the $\mathrm{Ni}$ atoms, $m_{\mathrm{DFT}}^{\mathrm{Ni}}$, and the $\mathrm{Cr}$ atoms, $m_{\mathrm{DFT}}^{\mathrm{Cr}}$, depend strongly on the sum of the moments of their Fe first nearest neighbors (1nn). This is illustrated in Fig. 2, which represents the relaxed moment obtained from a magnetic configuration for which the initial moments were initialized in the AFM1 state. Furthermore, this result is quite insensitive to the initial magnetic configuration. The trends are clear: $\mathrm{Cr}$ atoms tend to be antiferromagnetic with respect to the $1 \mathrm{nn}$ Fe moments, as is the case in bcc Fe-Cr alloys, ${ }^{50}$ and their local magnetic moments range from $-1.5 \mu_{B}$ to $1.5 \mu_{B}$. Conversely, the $\mathrm{Ni}$ moments are ferromagnetic with respect to the $1 \mathrm{nn} \mathrm{Fe}$ moments, as is the case in bcc Fe-Ni alloys, ${ }^{51,52}$ and their local magnetic moments range from $-0.4 \mu_{B}$ to $0.4 \mu_{B}$. It has to be noted that $\mathrm{Fe}$ forms $70 \%$ of our model alloy and therefore many Fe-rich environments exist. Thus, as the magnetic moments of $\mathrm{Fe}$ atoms range between $-2 \mu_{B}$ and $2 \mu_{B}$, as we will see next, it is not surprising that the sums of the moments of Fe 1nn displayed in Fig. 2 lie between $-10 \mu_{B}$ and $10 \mu_{B}$. By contrast, the behavior of Fe moments is less predictable, as the magnitude of Fe moments is only slightly ruled by its local chemistry. These results are consistent with the work published by James et al. ${ }^{52}$ who found that in binary alloys, the Fe moment is mostly correlated with its chemical surrounding, while the Ni moment depends more on the magnetic surrounding.
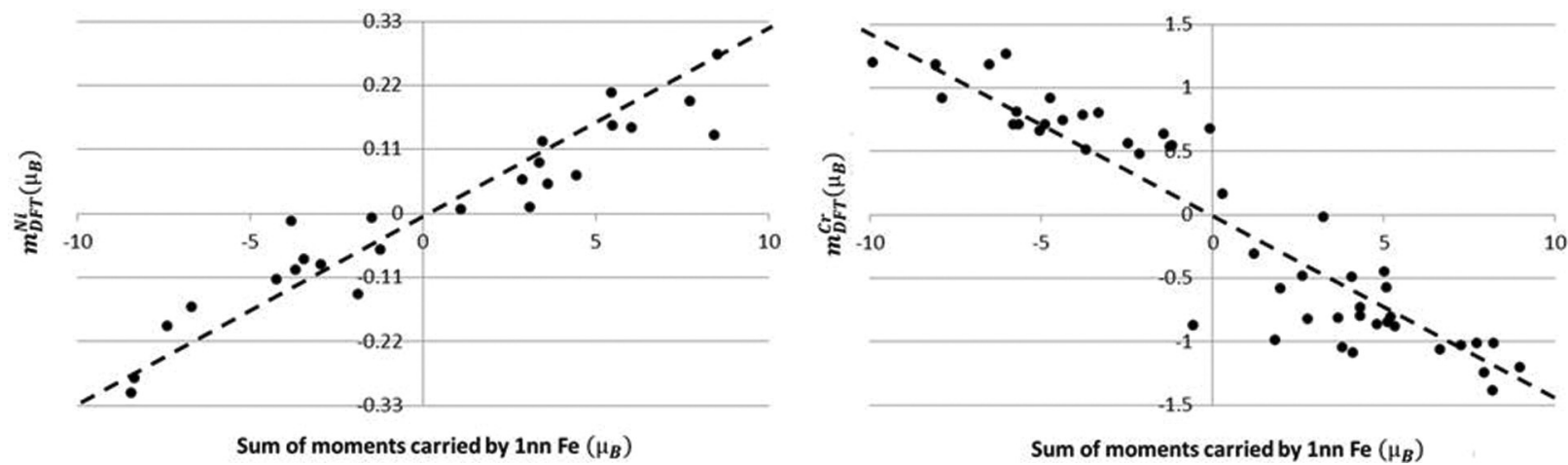

FIG. 2. Magnitude of relaxed moments carried by $\mathrm{Cr}$ atoms, $m_{\mathrm{DFT}}^{\mathrm{Cr}}$, versus the sum of the moments of their Fe 1nn (left figure). Magnitude of relaxed moments carried by $\mathrm{Ni}$ atoms, $m_{\mathrm{DFT}}^{\mathrm{Ni}}$, versus the sum of the moments of their Fe $1 \mathrm{nn}$ (right figure). The moments were initialized in the AFM1 state. 
Finally, a number of moment flips occur during the course of the calculation, in particular when starting with a PM phase. In that case numerous local moment flips occurred during the relaxation, inducing important changes of the magnetic interactions felt at each lattice site, leading to multiple relaxed magnetic structures. The total energy variation associated with these changes was found to be less than $1 \mathrm{meV}$ per atom on average. These moment changes even happened when using atomic positions and local moments of an already relaxed configuration as input parameter to restart a second run. During the course of the first calculation the local moments of about $10 \%$ of the $\mathrm{Fe}$ atoms decrease and lie between -1 and $1 \mu_{B}$ ("low spin Fe atoms"). These local moments are then subjected to significant changes when restarting the first calculation. This behavior is certainly due to the competition between many magnetic states close in energy, thus making difficult the use of the PM phase as the reference state for point defect calculations, although it would have been appropriate for this study. For all the above reasons, the AFM1 state was selected to calculate the point defect formation energies. We found an fcc phase with lattice parameter of $3.52 \AA$ when using the CL method and an fct phase with lattice parameters of $a=3.50 \AA$, $b=3.50 \AA$, and $c=3.57 \AA$ when using the FR method.

In a recent paper by Ekholm and Abrikosov for an alloy in the same electron concentration range, fcc Fe-Mn, ${ }^{53}$ limitations of the current functionals were analyzed, and the necessity to compare theory with experiment in terms of lattice parameter was underlined as an essential indicator of the accuracy of calculations. Although no experimental lattice parameters are available in the fcc Fe-10Ni-20Cr system, lattice parameters of various $\mathrm{Fe}-\mathrm{Ni}-\mathrm{Cr}$ alloys (with $30 \%-67 \%$ $\mathrm{Ni}, 1 \%-51 \% \mathrm{Fe}$ and $17 \%-32 \% \mathrm{Cr}$ ) are summarized by Marruco and range between 3.52 and $3.59 \AA$ after long-term exposures at temperatures between $450{ }^{\circ} \mathrm{C}$ and $600^{\circ} \mathrm{C} .{ }^{54}$ However, these lattice parameters have to be scaled down to $0 \mathrm{~K}$ to compare to DFT. For that purpose we used as a rule the thermal expansion found by Hayase et al. in $\mathrm{Fe}-\mathrm{Ni}$ binaries. ${ }^{55} \mathrm{We}$ found that the predicted lattice parameter is about $1 \%$ underestimated using this rough approximation. It can be mentioned that this underestimation could be larger in fcc Fe-10Ni-20Cr because the experimental lattice parameter is expected to increase linearly with increasing $\mathrm{Cr}$ and $\mathrm{Fe}$ concentrations. ${ }^{54} \mathrm{We}$ also find that the predicted lattice parameter is about $1 \%$ underestimated, comparing to a number of austenitic grades $(301,304,309,316$, and 4306 steels) close in chemical composition ${ }^{56}$ and using the thermal expansion of 304 as a rule. The same DFT underestimation is found for bcc $\mathrm{Fe}(-1.2 \%)$ and is thus expected and acceptable.

\section{POINT DEFECT CALCULATIONS}

The substitution energy calculations performed in the AFM1 reference state using DFT, $\Delta^{B \rightarrow A} \mu$, and used to obtain an estimate of zero Kelvin reference energies for the addition

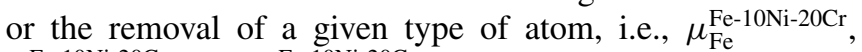
$\mu_{\mathrm{Ni}}^{\mathrm{Fe}-10 \mathrm{Ni}-20 \mathrm{Cr}}$, and $\mu_{\mathrm{Cr}}^{\mathrm{Fe}-10 \mathrm{Ni}-20 \mathrm{Cr}}$ [from Eq. (4) to Eq. (6)], are listed in Table I. The difference between substitution energies obtained in the alloy, $\mu_{A}^{\mathrm{Fe}-10 \mathrm{Ni}-20 \mathrm{Cr}}$, and in the pure elements, $\mu_{A}^{0}$, are presented in Table II. It can be noted that reference chemical potentials calculated in the AFM1 Fe-10Ni-20Cr
TABLE I. Minimum values of substitution energies, $\Delta^{B \rightarrow A} \mu(A$ and $B$ refer to the element type), performed to obtain an estimate of zero Kelvin reference energies in DFT for the addition or the removal of a given type of atom in the AFM1 Fe-10Ni-20Cr system, i.e., $\mu_{\mathrm{Fe}}^{\mathrm{Fe}-10 \mathrm{Ni}-20 \mathrm{Cr}}, \mu_{\mathrm{Ni}}^{\mathrm{Fe}-10 \mathrm{Ni}-20 \mathrm{Cr}}$, and $\mu_{\mathrm{Cr}}^{\mathrm{Fe}-10 \mathrm{Ni}-20 \mathrm{Cr}}$, are presented along with the number of calculations performed.

Substitution energy (eV) Number of calculations Minimum value

\begin{tabular}{lrr}
\hline$\Delta \mu^{\mathrm{Fe} \rightarrow \mathrm{Ni}}$ & 40 & 2.50 \\
$\Delta \mu^{\mathrm{Ni} \rightarrow \mathrm{Fe}}$ & 26 & -2.81 \\
$\Delta \mu^{\mathrm{Fe} \rightarrow \mathrm{Cr}}$ & 40 & -1.43 \\
$\Delta \mu^{\mathrm{Cr} \rightarrow \mathrm{Fe}}$ & 40 & 0.86 \\
$\Delta \mu^{\mathrm{Ni} \rightarrow \mathrm{Cr}}$ & 26 & -4.07 \\
$\Delta \mu^{\mathrm{Cr} \rightarrow \mathrm{Ni}}$ & 40 & 3.49 \\
\hline
\end{tabular}

reference state, $\mu_{A}^{\mathrm{Fe}-10 \mathrm{Ni}-20 \mathrm{Cr}}$, never exceed their associated bulk chemical potentials, $\mu_{A}^{0}$. This result indicates that all elements are more stable in the $\mathrm{Fe}-10 \mathrm{Ni}-20 \mathrm{Cr}$ reference state than in their respective pure phases of lowest energies found using DFT $\left(\mu_{A}^{\mathrm{Fe}-10 \mathrm{Ni}-20 \mathrm{Cr}}-\mu_{A}^{0}<0\right.$ for all elements $\left.A\right)$.

It has to be mentioned again that the estimates of chemical potentials $\mu_{A}^{\mathrm{Fe}-10 \mathrm{Ni}-20 \mathrm{Cr}}$ presented in Table II only represent zero Kelvin reference energies in DFT for the addition or the removal of a given type of atom in our AFM1 Fe-10Ni$20 \mathrm{Cr}$ reference configuration. In any case, the use of these estimated chemical potentials will only cause a global shift in the formation energy values thus obtained, with respect to using the exact values, and will not change the trends observed and the relationship of the formation energies with the point defect local environment.

Point defect formation energies, calculated using the chemical potential estimates (Table II) are presented in Table III. This table presents for each set of formation energies, the number of calculations performed, the mean value, the energy range, and the reference state considered. Our results can be compared with the formation energy values found by Klaver et al. in dilute $\mathrm{Fe}-\mathrm{Ni}-\mathrm{Cr}$ austenitic alloys ${ }^{23}$ where, especially for the dumbbell formation energies, significant differences are observed. Indeed, the mean value found for the vacancy formation energies is similar in the fct AFM1 $\gamma$-Fe system and in our model alloy while dumbbell formation is found to be significantly higher in the fct AFM $1 \gamma$-Fe. It can, however, be noted that the same general order of preference for the different alloying elements can be found in dumbbell sites. Indeed, $\mathrm{Fe}-\mathrm{Fe}$ dumbbells are found to be the most stable. $\mathrm{Ni}$ is very unlikely to be found in mixed Fe-Ni dumbbells, Ni-Ni dumbbells are even more unlikely to form, whereas $\mathrm{Cr}$, on the other hand, can form mixed dumbbells. These results are

TABLE II. Difference between the reference chemical potentials calculated using DFT, in a pure fcc system, $\mu_{A}^{0}$, and those calculated in the AFM1 Fe-10Ni-20Cr system, $\mu_{A}^{\mathrm{Fe}-10 \mathrm{Ni}-20 \mathrm{Cr}}$. The magnetic ordering considered for the calculations were the AFM1, FM, and nonmagnetic phases in pure systems (i.e., the lowest energy states) of $\mathrm{Fe}, \mathrm{Ni}$, and $\mathrm{Cr}$, respectively.

\begin{tabular}{lccc}
\hline \hline Alloying elements & $\mathrm{Fe}$ & $\mathrm{Ni}$ & $\mathrm{Cr}$ \\
\hline$\mu_{A}^{\mathrm{Fe}-10 \mathrm{Ni}-20 \mathrm{Cr}}-\mu_{A}^{0}(\mathrm{eV})$ & -0.07 & -0.002 & -0.249 \\
\hline \hline
\end{tabular}


TABLE III. Energy range found for each set of formation energy calculations is presented along with the mean value, the number of calculation, and the reference state used. For instance, $\Delta E_{\mathrm{DFT}}^{V}$ refers to the vacancy formation energy.

\begin{tabular}{llccc}
\hline \hline $\begin{array}{l}\text { Reference } \\
\text { state }\end{array}$ & $\begin{array}{c}\text { Point defect } \\
\text { formation } \\
\text { energy }(\mathrm{eV})\end{array}$ & $\begin{array}{c}\text { Number of } \\
\text { calculations }\end{array}$ & $\begin{array}{c}\text { Energy } \\
\text { range }(\mathrm{eV})\end{array}$ & $\begin{array}{c}\text { Mean } \\
\text { value }(\mathrm{eV})\end{array}$ \\
\hline fctAFM1 & $\Delta E_{\mathrm{DFT}}^{V}$ & 60 & $1.76-2.19$ & 1.96 \\
& $\Delta E_{\mathrm{DFT}}^{[100] \mathrm{Fe}-\mathrm{Fe}}$ & 60 & $2.61-3.34$ & 3.02 \\
& $\Delta E_{\mathrm{DFT}}^{[010] \mathrm{Fe}-\mathrm{Fe}}$ & 40 & $2.72-3.50$ & 3.06 \\
& $\Delta E_{\mathrm{DFT}}^{[100] \mathrm{Fe}-\mathrm{Ni}}$ & 60 & $2.81-3.80$ & 3.37 \\
& $\Delta E_{\mathrm{DFT}}^{[010] \mathrm{Fe}-\mathrm{Ni}}$ & 40 & $3.12-3.96$ & 3.48 \\
& $\Delta E_{\mathrm{DFT}}^{[100] \mathrm{Fe}-\mathrm{Cr}}$ & 40 & $2.68-3.31$ & 3.05 \\
& $\Delta E_{\mathrm{DFT}}^{[010] \mathrm{Fe}-\mathrm{Cr}}$ & 40 & $2.76-3.41$ & 3.04 \\
& $\Delta E_{\mathrm{DFT}}^{[100] \mathrm{Ni}-\mathrm{Ni}}$ & 40 & $323-3.95$ & 3.65 \\
& $\Delta E_{\mathrm{DFT}}^{[100] \mathrm{Cr}-\mathrm{Cr}}$ & 40 & $2.97-3.71$ & 3.31 \\
& $\Delta E_{\mathrm{DFT}}^{[100] \mathrm{Ni}-\mathrm{Cr}}$ & 40 & $2.94-3.79$ & 3.35 \\
fctAFM1 & $\Delta E_{\mathrm{DFT}}^{[100] \mathrm{Fe}-\mathrm{Fe}}$ & 40 & $2.75-3.51$ & 3.05 \\
& $\Delta E_{\mathrm{DFT}}^{[010] \mathrm{Fe}-\mathrm{Fe}}$ & 40 & $2.79-3.56$ & 3.05 \\
\hline \hline
\end{tabular}

not in agreement with the experimentally derived size factors for $\mathrm{Ni}$ and $\mathrm{Cr}$ solutes in austenitic SS type 316 and in the pure materials ${ }^{57}$ for which $\mathrm{Ni}$ is found to be undersized. These results are not, however, necessarily in contradiction since the experimental results were obtained at high temperatures. Indeed, it is worth reminding that at temperatures at which nuclear reactors operate, $\mathrm{Fe}-\mathrm{Ni}-\mathrm{Cr}$ alloys are paramagnetic, whereas only the AFM1 state was found to be reliably usable for DFT calculations. This seems to indicate that volume elastic effects which are prominent at high temperatures become negligible relative to the magnetic energies at $0 \mathrm{~K}$.

We also obtained a large energy range for each set of point defect formations, thus underlying the key role played by the local environment. In particular, Fig. 3 illustrates the influence of the number of $\mathrm{Ni}$ and $\mathrm{Cr} 1 \mathrm{nn}$ to the vacancy on the formation energy values $E_{f}^{V}$. Indeed, vacancies prefer Ni-rich and $\mathrm{Cr}$ poor environments.
TABLE IV. Variable notations used to describe the local environment surrounding the point defects are represented along with the number of their atomic sites. $1 \mathrm{nn} \_A$ variable refers to the number of $A$ atoms first nearest neighbors to the point defect. 1nnc_A and 1nnt_A variables refer to the number of $A$ atoms positioned in compressive and in tensile sites, respectively. $1 \mathrm{nncFe} \_A$ and $1 \mathrm{nncS} \_A$ variables refer to the number of $A$ atoms positioned in compressive sites, located closer to the Fe atom of the dumbbell and closer to the solute atom of the dumbbell, respectively.

\begin{tabular}{lc}
\hline \hline Local variables & Number \\
\hline $1 \mathrm{nn} \_A$ & 12 \\
$1 \mathrm{nnc} \_A$ & 8 \\
$1 \mathrm{nncFe} \_A$ & 4 \\
$1 \mathrm{nncS} A A$ & 4 \\
$1 \mathrm{nnt} A A$ & 4 \\
\hline \hline
\end{tabular}

The influence of the content of the point defect neighbor shells was investigated in more detail and it was found that the point defect formation energies could be estimated using linear regressions (LRs). The variables considered are listed in Table IV. The simplest set of variables describing the atomic arrangement surrounding the point defects is the one which only considers the number of alloying elements positioned in their nearest shells. We considered in this work the $1 \mathrm{nn}$ of the point defect center of mass. They will be referred to in the next sections, as $1 \mathrm{nn} \_A$, where $A$ indicates the chemical type of the neighbor considered. In contrast to the vacancy, the specific orientation of the dumbbell induces anisotropic relaxations for atoms belonging to its first shells. We have thus considered two different variables to describe the first nearest atoms of a dumbbell defect, by introducing in the notation " $t$ " when the atom is positioned in a tensile site or "c" when the atom is positioned in a compressive site. $1 \mathrm{nnc} \_\mathrm{Ni}$ thus refers to a $\mathrm{Ni}$ atom which is situated at $1 \mathrm{nn}$ separation of a dumbbell and in a compressive site, i.e., one of the black spheres in Fig. 4. It also can be assumed that for both $\mathrm{Fe}-\mathrm{Ni}$ and $\mathrm{Fe}-\mathrm{Cr}$ mixed dumbbells, first nearest atoms positioned in compressive sites may behave differently when located closer to the $\mathrm{Fe}$ atom than the solute atom of the mixed dumbbell. These two different kinds of compressive sites have been therefore distinguished

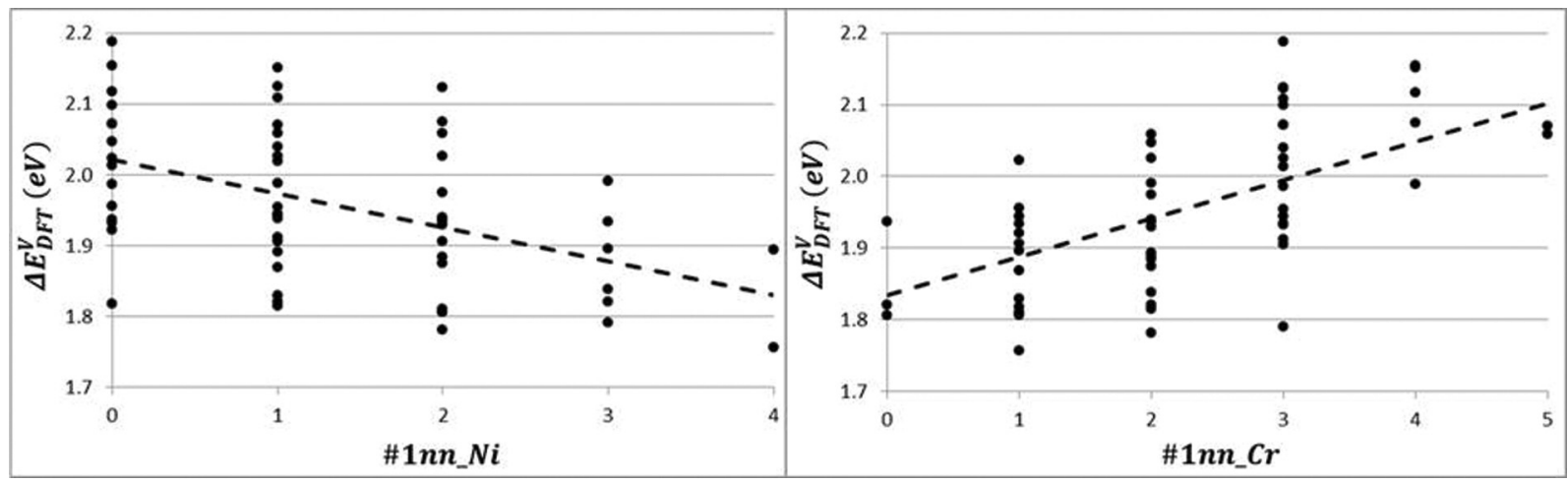

FIG. 3. Evolution of the vacancy formation energy $\Delta E_{\mathrm{DFT}}^{V}$ as a function of the number of nearest-neighbor Ni atoms surrounding the vacancy $1 \mathrm{nn} \_\mathrm{Ni}$ (left figure). Evolution of the vacancy formation energy $\Delta E_{\mathrm{DFT}}^{V}$ as a function of the number of nearest-neighbor Cr atoms surrounding the vacancy $1 \mathrm{nn} \_\mathrm{Cr}$ (right figure). These figures display the results for the 60 vacancy configurations performed in the fct AFM1 reference state. 


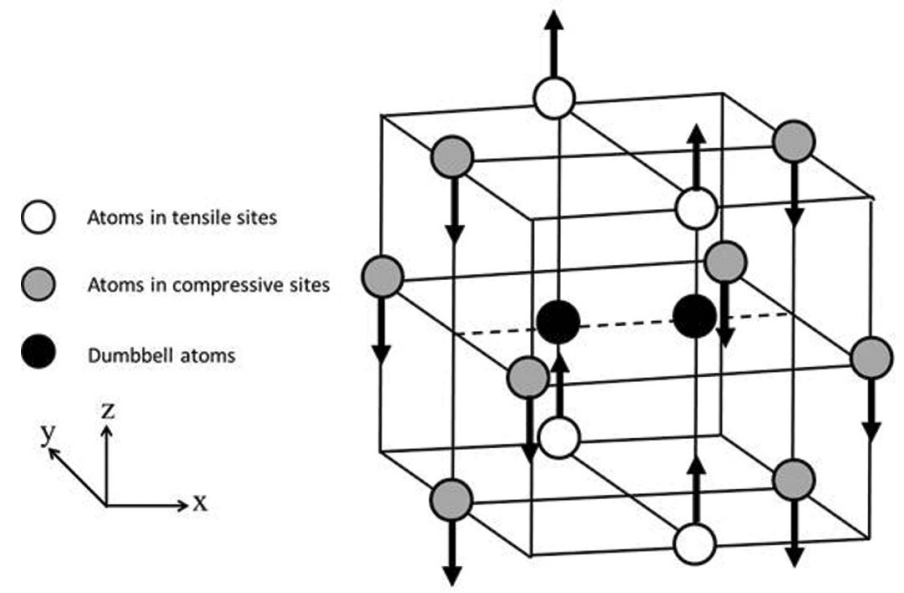

(a)

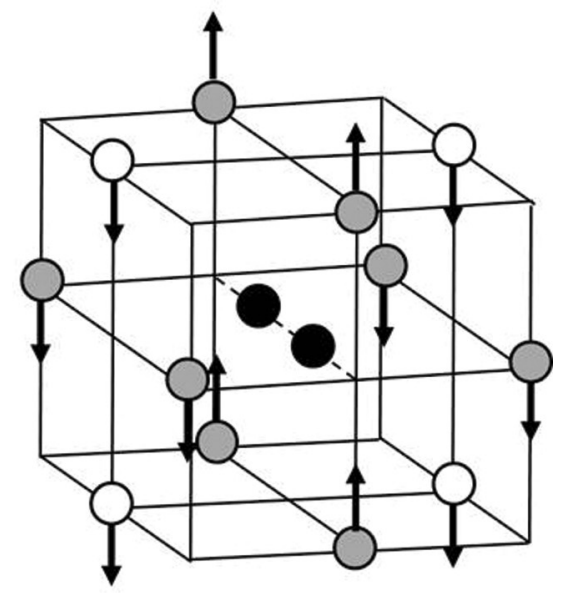

(b)

FIG. 4. Representation of atoms at $1 \mathrm{nn}$ separation to a dumbbell defect oriented either along the [100] direction (a) or along the [010] direction (b), in a fcc lattice. Atoms positioned in compressive sites are represented in gray and atoms in tensile sites are represented in white. Dumbbell atoms are represented in black. Arrows indicate the local moments in both figures and the magnetic planes of the AFM1 reference state are shown to aid visualization.

by introducing in the variable notation either "Fe" or "S," respectively, when they were positioned in the plane near either the Fe or the solute atom of the mixed dumbbell. The $1 \mathrm{nncFe}$ Ni variable thus refers to the number of $\mathrm{Ni}$ atoms positioned in compressive sites, located closer to the Fe atom of the mixed dumbbell. No such differentiation was considered for tensile sites since they are equidistant from the two atoms which form the dumbbell.

A systematic study has been performed in order to determine which variables among those listed in Table IV have the greatest weight in the estimation of the formation energies calculated using DFT. The results for each point defect are summarized in Table $\mathrm{V}$ which presents the best estimates of formation energies using LRs. The adequacy between the formation energies calculated using DFT, $\Delta E_{\mathrm{DFT}}$, and their estimated values using multiple LRs, $\Delta E_{\mathrm{LR}}$, was determined using the Pearson product-moment correlation coefficient $r_{\Delta E_{\mathrm{DFT}} \Delta E_{\mathrm{LR}}}$. Correlation coefficients (CCs) were obtained by dividing the covariance of the two variables, $\Delta E_{\mathrm{DFT}}$ and $\Delta E_{\mathrm{LR}}$, by the product of their standard deviations as follows:

$$
\begin{aligned}
& r_{\Delta E_{\mathrm{DFT}} \Delta E_{\mathrm{LR}}} \\
& =\frac{\sum_{k=1}^{n}\left(\Delta E_{\mathrm{DFT}}^{k}-\overline{\Delta E_{\mathrm{DFT}}^{k}}\right)\left(\Delta E_{\mathrm{LR}}^{k}-\overline{\Delta E_{\mathrm{LR}}^{k}}\right)}{\sqrt{\sum_{k=1}^{n}\left(\Delta E_{\mathrm{DFT}}^{k}-\overline{\Delta E_{\mathrm{DFT}}^{k}}\right)^{2} \sum_{i=1}^{n}\left(\Delta E_{\mathrm{LR}}^{k}-\overline{\Delta E_{\mathrm{LR}}^{k}}\right)^{2}}},
\end{aligned}
$$

where $n$ refers to the number of points considered. This relation measures the strength of the linear relationship between the two sets of data $\Delta E_{\mathrm{DFT}}^{k}$ and $\Delta E_{\mathrm{LR}}^{k}$, respectively. The Pearson correlation is +1 in the case of a perfect positive linear relationship and -1 in the case of a perfect negative linear relationship. For intermediate values, the more the Pearson

TABLE V. LRs of interest used to estimate the required energies $\Delta E$ (in $\mathrm{eV}$ ) to introduce a point defect at different lattice sites of the AFM1 reference state using DFT, i.e., $\Delta E_{\mathrm{DFT}}^{V}$ and $\Delta E_{\mathrm{DFT}}^{\mathrm{Fe}-A}$ ( $A$ refers to the element type) energies. The obtained parameter values assigned to the variables displayed at the top of the rows are summarized for each LR along with the crystallographic phase and the LR type which indicates which DFT data set was fitted. Variables considered, i.e., $1 \mathrm{nn} \_A, 1 \mathrm{nnc} \_A, 1 \mathrm{nnt} \_A, 1 \mathrm{nncS} \_A$, and $1 \mathrm{nncFe} \_A$ ( $A$ refers to the element type), are listed in Table IV. For instance, the best estimate for the formation energy of the [100] dumbbell in the fct phase is obtained by the

\begin{tabular}{|c|c|c|c|c|c|c|c|c|c|c|c|c|c|}
\hline LR type & Phase & 1nn_Ni & 1nn_Cr & 1nnc_Ni & 1nnc_Cr & $1 \mathrm{nncFe} \_\mathrm{Ni}$ & 1nncFe_Cr & 1nncS_Ni & 1nncS_Cr & 1nnt_Ni & 1nnt_Cr & Cst & $\mathrm{CC}$ \\
\hline$\Delta E_{\mathrm{LR}}^{V}$ & fct & -0.04 & 0.05 & & & & & & & & & 2.00 & 0.74 \\
\hline$\Delta E_{\mathrm{LR}}^{[100] \mathrm{Fe}-\mathrm{Fe}}$ & fct & & & 0.08 & -0.11 & & & & & -0.05 & -0.07 & 3.00 & 0.75 \\
\hline$\Delta E_{\mathrm{LR}}^{[010] \mathrm{Fe}-\mathrm{Fe}}$ & fct & & & 0.15 & -0.06 & & & & & -0.04 & -0.07 & 3.05 & 0.82 \\
\hline$\Delta E_{\mathrm{LR}}^{[100] \mathrm{Fe}-\mathrm{Fe}}$ & $\mathrm{fcc}$ & & & 0.10 & -0.08 & & & & & -0.01 & -0.06 & 3.05 & 0.65 \\
\hline$\Delta E_{\mathrm{LR}}^{[010] \mathrm{Fe}-\mathrm{Fe}}$ & fcc & & & 0.14 & -0.05 & & & & & -0.05 & -0.07 & 3.05 & 0.75 \\
\hline$\Delta E_{\mathrm{LR}}^{[100] \mathrm{Fe}-\mathrm{Ni}}$ & fct & & & & & 0.03 & -0.14 & -0.08 & -0.08 & & & 3.40 & 0.70 \\
\hline$\Delta E_{\mathrm{LR}}^{[010] \mathrm{Fe}-\mathrm{Ni}}$ & fct & & & & & 0.12 & -0.10 & 0.03 & -0.09 & & & 3.50 & 0.70 \\
\hline$\Delta E_{\mathrm{LR}}^{[100] \mathrm{Fe}-\mathrm{Cr}}$ & fct & & & & & 0.02 & -0.08 & 0.01 & 0.08 & & & 3.05 & 0.57 \\
\hline$\Delta E_{\mathrm{LR}}^{[010] \mathrm{Fe}-\mathrm{Cr}}$ & fct & & & & & 0.13 & -0.05 & 0.11 & 0.12 & & & 3.05 & 0.79 \\
\hline
\end{tabular}
formula $\Delta E_{\mathrm{LR}}^{[100] \mathrm{Fe}-\mathrm{Fe}}=0.08 \times 1 \mathrm{nnc} \_\mathrm{Ni}-0.11 \times 1 \mathrm{nnc} \_\mathrm{Cr}-0.05 \times 1 \mathrm{nnt} \_\mathrm{Ni}-0.07 \times 1 \mathrm{nnt} \_\mathrm{Cr}+\mathrm{Cst}$, where Cst in this precise case is 3.0 . 


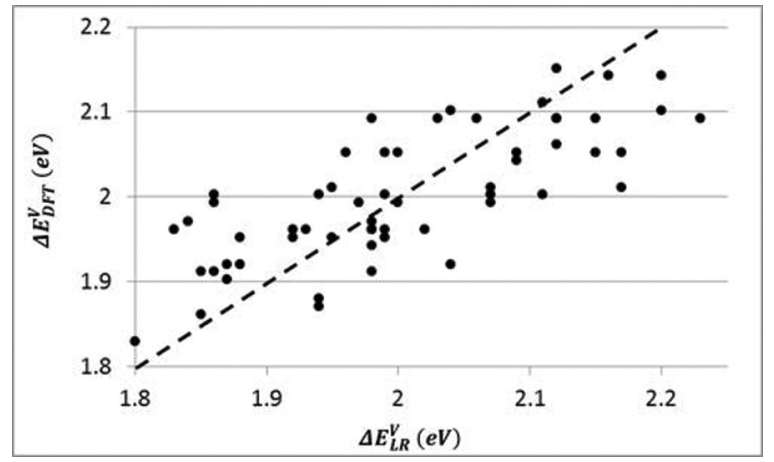

FIG. 5. Vacancy formation energies (in $\mathrm{eV}$ ) obtained using DFT, $\Delta E_{\mathrm{DFT}}^{V}$, compared with their values estimated using LR 1 in Table V, i.e., $\Delta E_{\mathrm{LR}}^{V}=-0.04 \times 1 \mathrm{nn} \_\mathrm{Ni}+0.05 \times 1 \mathrm{nn} \_\mathrm{Cr}+2.0$, where 1nn_Ni is the number of Ni $1 \mathrm{nn}$ and $1 \mathrm{nn} \_\mathrm{Cr}$ is the number of $\mathrm{Cr} 1 \mathrm{nn}$ to the vacancy. A CC of 0.74 is obtained between these two sets of energies.

correlation approaches the zero value, the less correlation exists between the two sets of data $\Delta E_{\mathrm{DFT}}^{k}$ and $\Delta E_{\mathrm{LR}}^{k}$, respectively.

\section{A. Vacancy formation energies}

The parameter values presented for $\Delta E_{\mathrm{LR}}^{V}$ in Table $\mathrm{V}$ refers to the vacancy formation energy. It can be seen that a LR which considers only the number of $\mathrm{Ni}$ and $\mathrm{Cr}$ atoms $1 \mathrm{nn}$ to the vacancy (1nn_Cr and $1 \mathrm{nn} \_N i$ variables), was sufficient to obtain a good value of 0.74 for the CC. Parameter values assigned to these variables have a negative sign for $\mathrm{Ni}$ and a positive sign for $\mathrm{Cr}$, showing that to a first approximation, it costs on average, a higher energy to introduce a vacancy near $\mathrm{Cr}$ than Ni 1nn atoms. The correlation existing between these two sets of energies can be seen in Fig. 5. These results are in strong agreement with the work of Klaver et al. in which $\mathrm{Ni}$ was found to bind to vacancies, while $\mathrm{V}-\mathrm{Cr}$ interactions were repulsive. ${ }^{23}$ Although the neighboring $\mathrm{Fe}$ atoms impose the moment carried by both $\mathrm{Cr}$ and $\mathrm{Ni}$ atoms as shown in Fig. 2, no dependence of the evolution of the vacancy formation energy was found with the first nearest atoms of Fe. Better estimates can be obtained by performing LRs considering more variables. In particular, we obtained an improved $\mathrm{CC}$ value of 0.80 using the four-parameter LR which takes into account the number of $\mathrm{Cr}$ atoms in the first three shells and the number of $1 \mathrm{nn}$ Ni surrounding the vacancy.

It can be mentioned that substitutional disordered fcc $\mathrm{Fe}-\mathrm{Ni}-\mathrm{Cr}$ alloys in the paramagnetic state were studied by Delczeg $^{30}$ using CPA, i.e., by neglecting the local lattice relaxation and short range order. As forces are not calculated in this approach, the vacancy relaxation configuration was obtained by minimizing the energy as a function of the distance between the first nearest neighbors of the vacancy and of the supercell volume only. For Fe-10Ni-20Cr, the relaxed vacancy formation energy was found to be $1.95 \mathrm{eV}$. The results were also interpreted in terms of effective chemical potentials, and the theoretical predictions obtained for homogeneous chemistry and relaxed nearest-neighbor lattice sites are in line with the experimental observations as well as our results.
Indeed, $\mathrm{Ni}$ was found to decrease and $\mathrm{Cr}$ to increase the vacancy formation energy of this ternary system.

\section{B. Fe-Fe dumbbell formation energies}

Similarly to what has been done in the case of vacancies, LRs of the DFT data were performed for each dumbbell studied. However, in the case of $\mathrm{Fe}-\mathrm{Fe}$ dumbbells, we also considered the number of atoms in tensile and in compressive sites. In this section, the DFT formation energies of Fe$\mathrm{Fe}$ dumbbells oriented along the [100] axis, $\Delta E_{\mathrm{DFT}}^{[100] \mathrm{Fe}-\mathrm{Fe}}$, and along the [010] axis, $\Delta E_{\mathrm{DFT}}^{[010] \mathrm{Fe}-\mathrm{Fe}}$, are compared to their best estimates using LRs $\Delta E_{\mathrm{LR}}^{[100] \mathrm{Fe}-\mathrm{Fe}}$ and $\Delta E_{\mathrm{LR}}^{[010] \mathrm{Fe}-\mathrm{Fe}}$, respectively, in Table V. The LRs were performed in both fcc and fct AFM1 reference states to analyze the impact of the calculation method on results.

The results obtained in the fct phase show first that, as in the case of the vacancy, the knowledge of the number of $\mathrm{Cr}$ and $\mathrm{Ni} 1 \mathrm{nn}$ to the $\mathrm{Fe}-\mathrm{Fe}\langle 100\rangle$ dumbbell allows a correct estimate of the formation energies. However, the best estimates were obtained when distinguishing between $\mathrm{Cr}$ and $\mathrm{Ni}$ atoms positioned in tensile sites, from those positioned in compressive sites. The LRs presented in Table $\mathrm{V}$ provide estimates of $\Delta E_{\mathrm{DFT}}^{[100] \mathrm{Fe}-\mathrm{Fe}}$ and $\Delta \mathrm{E}_{\mathrm{DFT}}^{[010] \mathrm{Fe}-\mathrm{Fe}}$ energies with $\mathrm{CC}$ values of, respectively, 0.75 and 0.82 in the fct phase. This proves the different role played by atoms positioned in tensile and compressive sites. The corresponding correlation existing between the DFT values $\Delta E_{\mathrm{DFT}}^{[100] \mathrm{Fe}-\mathrm{Fe}}$ and their estimated values $\Delta E_{\mathrm{LR}}^{[100] \mathrm{Fe}-\mathrm{Fe}}$ can be seen in Fig. 6.

The different parameter values obtained for these LRs confirm that both $\mathrm{Cr}$ and $\mathrm{Ni}$ atoms positioned in tensile and compressive sites do not contribute equally to the $\langle 100\rangle$ $\mathrm{Fe}-\mathrm{Fe}$ formation energy. The most prominent element proving this feature is the negative parameter values obtained for the $1 \mathrm{nnt} \_\mathrm{Ni}$ variable, whereas the parameter values assigned to the 1nnc_Ni variable are positive. Furthermore, the relative

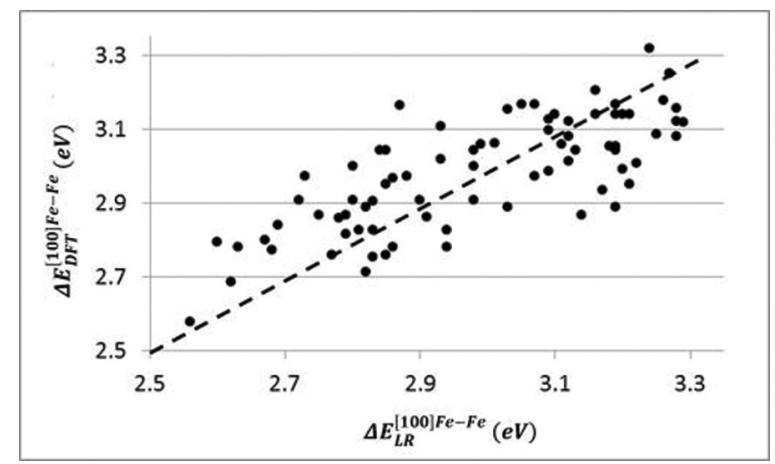

FIG. 6. Formation energies (in $\mathrm{eV}$ ) of $\mathrm{Fe}-\mathrm{Fe}$ dumbbells oriented along the [100] direction obtained using DFT, $\Delta E_{\mathrm{DFT}}^{[100] \mathrm{Fe}-\mathrm{Fe}}$, compared to their values estimated using the LR 2 in Table V, i.e., $\Delta E_{\mathrm{LR}}^{[100] \mathrm{Fe}-\mathrm{Fe}}=0.08 \times 1 \mathrm{nnc} \_\mathrm{Ni}-0.11 \times 1 \mathrm{nnc} \_\mathrm{Cr}-$ $0.05 \times 1 \mathrm{nnt} \_\mathrm{Ni}-0.07 \times 1 \mathrm{nnt} \_\mathrm{Cr}+3.0$. $1 \mathrm{nnc} \_\mathrm{Ni}$ and $1 \mathrm{nnc} \_\mathrm{Cr}$ variables correspond, respectively, to the number of $\mathrm{Ni}$ and $\mathrm{Cr}$ $1 \mathrm{nn}$, positioned in compressive sites. 1nnt_Ni and 1nnt_Cr variables correspond, respectively, to the number of $\mathrm{Ni}$ and $\mathrm{Cr} 1 \mathrm{nn}$, positioned in tensile sites. CC of 0.75 is obtained between these two sets of energies. 
importance between compressive and tensile sites was investigated by performing two-parameter LRs taking into account $\mathrm{Cr}$ and $\mathrm{Ni}$ atoms positioned either only in tensile or only in compressive sites. Better CC values of 0.70 and 0.79 were found in the latter case, i.e., by only considering compressive sites in the estimate of $\Delta E_{\mathrm{DFT}}^{[100] \mathrm{Fe}-\mathrm{Fe}}$ and $\Delta E_{\mathrm{DFT}}^{[010] \mathrm{Fe}-\mathrm{Fe}}$ energies, respectively, thus showing the stronger influence of compressive sites.

Unlike the case of vacancies, the parameter values presented in Table $\mathrm{V}$ show that it cost more energy to introduce $\mathrm{Fe}-\mathrm{Fe}\langle 100\rangle$ dumbbells near $\mathrm{Ni}$ atoms than near $\mathrm{Cr}$ atoms. These results are in agreement with previous findings on binding energies of single $\mathrm{Ni}$ and $\mathrm{Cr}$ solutes to $\langle 100\rangle \mathrm{Fe}-\mathrm{Fe}$ self-interstitial dumbbells, in pure fcc $\mathrm{Fe}$, where $\mathrm{Ni}$ and $\mathrm{Cr}$ in compressive sites generally exhibited negative and positive binding energies, respectively. ${ }^{23}$

The same general conclusions as those presented in this section for the fct phase can be drawn for calculations performed in the fcc phase. Furthermore, no important changes in the LR parameters are observed relative to those obtained in the fct phase, as can be seen in Table V. The constraint on the supercell does not seem to affect too strongly the relationship between the local environment and the point defect energies.

One interesting result to note is that the LRs mostly yield similar coefficients for dumbbells oriented differently, i.e., whether the Fe-Fe dumbbells are oriented along the [100] direction (perpendicular to the AFM1 magnetic planes) or along the [010] direction (parallel to the AFM1 magnetic planes). Indeed, the effective interactions associated to the LRs found for the $\mathrm{Ni}$ and $\mathrm{Cr}$ solutes with point defects are consistent with what would intuitively be expected of moderately oversized and undersized solutes, respectively. ${ }^{23}$ $\mathrm{Ni}$ binds to the vacancy and is repelled from the compressive sites of $\langle 100\rangle \mathrm{Fe}-\mathrm{Fe}$ dumbbells, whereas $\mathrm{Cr}$ atoms generally exhibit the opposite tendencies. It also can be mentioned that the parameter values found for $\Delta E_{\mathrm{LR}}^{[010] \mathrm{Fe}-\mathrm{Fe}}$ are all higher than the ones found for $\Delta E_{\mathrm{LR}}^{[100] \mathrm{Fe}-\mathrm{Fe}}$ in Table V. However, the CCs are not very sensitive to the exact choice of LR parameter values. Slight variations in the LR parameters render very similar CCs. Therefore, it is not straightforward to conclude on the significance of the LR parameters that are finally chosen.
Differences in the effective interactions can, however, be expected owing to the fact that the local magnetic environment felt by dumbbell atoms introduced in the reference state strongly depends on the dumbbell axis relative to the AFM1 magnetic planes. Indeed, whatever the dumbbell orientation, there are on average as many spin-up as spin-down 1nn atoms in the AFM1 state, as represented schematically in Fig. 4. However, significant differences in the magnetic moment distribution exist for the tensile and the compressive sites. This feature is illustrated in Fig. 7, which represents the magnetic moments of the Fe atoms 1nn to an Fe-Fe dumbbell plotted as a function of their ionic relaxations, i.e., the displacement the atoms underwent after the dumbbell was introduced. The ionic relaxations will be noted as $\delta d_{\mathrm{DFT}}^{[100] \mathrm{Fe}-\mathrm{Fe}}$ or $\delta d_{\mathrm{DFT}}^{[010] \mathrm{Fe}-\mathrm{Fe}}$ depending on whether the dumbbell was introduced along the [100] or the [010] axis. Note that the sign of the ionic relaxation is a simple way of visualizing whether the atom is in a compressive site or in a tensile one. These figures clearly show significant differences whether Fe-Fe dumbbells are oriented along the [100] direction or along the [010] direction. Depending on the orientation of the dumbbell axis, the $\mathrm{Fe}$ moments are not distributed equivalently between tensile and compressive sites. Indeed, when the dumbbell is introduced with its axis perpendicular to the AFM1 magnetic planes [Fig. 4(a)], moments on Fe atoms in tensile sites are positive, whereas moments on $\mathrm{Fe}$ atoms in compressive sites (all with negative values in the reference state) have mostly negative values (about two-thirds in Fig. 7) due to a significant number of moment flips induced by the introduction of the [100] dumbbells, as will be discussed later. However, when the dumbbell is introduced with its axis parallel to the AFM1 magnetic planes [FIG. 4(b)], moments on Fe atoms in tensile sites are negative, but moments on $\mathrm{Fe}$ atoms in compressive sites can be either positive or negative. This arises from the specific configuration of the AFM1 structure. Indeed, compressive sites lie within two planes "down," whereas tensile sites lie within a single plane "up" when interstitials are introduced with their axis perpendicular to the AFM1 magnetic planes, as can be seen in Fig. 4. Similarly, tensile sites lie within a single plane "down," whereas compressive sites lie within two planes having, on average, the same amount of Fe atoms with moments "up" and "down" when
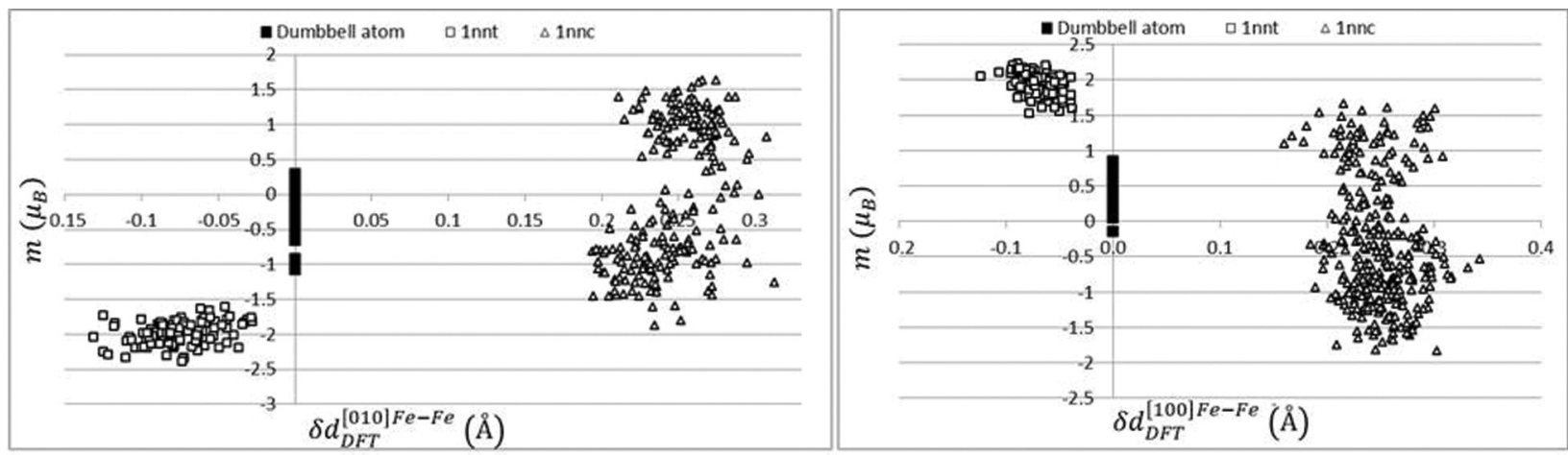

FIG. 7. Magnetic moments of Fe atoms $1 \mathrm{nn}$ to Fe-Fe dumbbells measured in the defect containing lattices $m$ as a function of their ionic relaxations, i.e., $\delta d_{\mathrm{DFT}}^{[100] \mathrm{Fe}-\mathrm{Fe}}$ or $\delta d_{\mathrm{DFT}}^{[010] \mathrm{Fe}-\mathrm{Fe}}$ depending on whether dumbbells were introduced along the [010] axis (left figure) or the [100] axis (right figure). Atoms in tensile sites (1nnt) are represented as open squares and atoms in compressive sites (1nnc) are represented with open triangles. Moments on Fe atoms of dumbbells are also represented along the zero abscissa (black squares). 

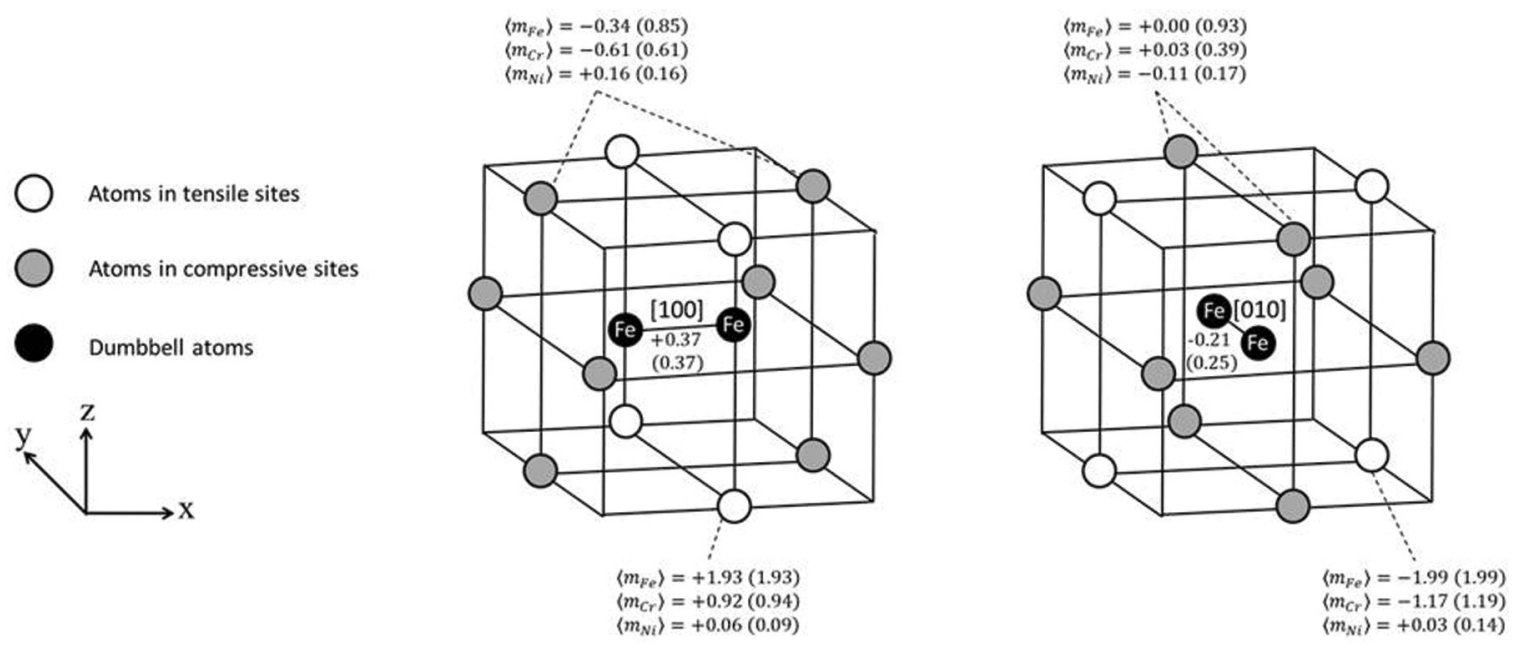

FIG. 8. Average values of DFT magnetic moments (in $\left.\mu_{B}\right),\langle m\rangle$, for atoms that are $1 \mathrm{nn}$ to $\langle 100\rangle \mathrm{Fe}-\mathrm{Fe}$ dumbbells. The values displayed in parentheses refer to the mean value of the absolute of moments.

interstitials are introduced with their axis parallel to the AFM1 magnetic planes. As a result, both $\mathrm{Cr}$ and $\mathrm{Ni}$ atom moments, which are imposed by $1 \mathrm{nn} \mathrm{Fe}$ atom moments as shown in Fig. 2, also present significant differences if the
Fe-Fe dumbbell is oriented along the [100] direction or along the [010] direction. The major trends can be seen schematically in Fig. 8, which presents, for the specific case of $\langle 100\rangle \mathrm{Fe}-\mathrm{Fe}$ dumbbells, the mean values of the magnetic moments carried

TABLE VI. Average values of DFT magnetic moments $\langle m\rangle$ for atoms that are $1 \mathrm{nn}$ to sites where defects were introduced in the reference state (without defect), and for atoms that are $1 \mathrm{nn}$ to defects (with defect). For each defect type, the relevant $1 \mathrm{nn}$ sites are considered: $1 \mathrm{nn}$ refers to first nearest-neighbor atoms; $1 \mathrm{nnc}$ and $1 \mathrm{nnt}$ refer to atoms positioned in compressive and in tensile sites, respectively; $1 \mathrm{nncFe}$ and $1 \mathrm{nncS}$ refer to atoms positioned in compressive sites, located closer to the Fe atom of the dumbbell and closer to the solute atom of dumbbells, respectively; and "Defect" refers to atoms positioned at defect sites. The values displayed in parentheses refer to the mean value of the absolute of moments.

\begin{tabular}{|c|c|c|c|c|c|c|c|}
\hline \multirow[b]{3}{*}{ Point defect type } & \multirow[b]{3}{*}{ Site type } & \multicolumn{3}{|c|}{$\langle m\rangle\left(\mu_{B}\right)$} & \multicolumn{3}{|c|}{$\langle m\rangle\left(\mu_{B}\right)$} \\
\hline & & \multicolumn{3}{|c|}{ Without defect } & \multicolumn{3}{|c|}{ With defect } \\
\hline & & $\mathrm{Fe}$ & $\mathrm{Cr}$ & $\mathrm{Ni}$ & $\mathrm{Fe}$ & $\mathrm{Cr}$ & $\mathrm{Ni}$ \\
\hline \multirow[t]{2}{*}{ Vacancy } & $1 \mathrm{nn}$ & -0.35 & -0.49 & 0.02 & -0.58 & -0.29 & -0.03 \\
\hline & Defect & 1.37 & & & & & \\
\hline \multirow[t]{3}{*}[100]{$\mathrm{Fe}-\mathrm{Fe}$} & $1 \mathrm{nnt}$ & 1.41 & 0.87 & 0.04 & $1.93(1.93)$ & $0.92(0.94)$ & $0.06(0.09)$ \\
\hline & $1 \mathrm{nnc}$ & -1.29 & -0.89 & 0.13 & $-0.34(0.85)$ & $-0.61(0.61)$ & $0.16(0.16)$ \\
\hline & Defect & 1.37 & & & $0.37(0.37)$ & & \\
\hline \multirow[t]{3}{*}[010]{$\mathrm{Fe}-\mathrm{Fe}$} & $1 \mathrm{nnt}$ & -1.23 & -0.90 & 0.09 & $-1.99(1.99)$ & $-1.17(1.19)$ & $0.03(0.14)$ \\
\hline & $1 \mathrm{nnc}$ & 0.05 & -0.19 & -0.05 & $0.00(0.93)$ & $0.03(0.39)$ & $-0.11(0.17)$ \\
\hline & Defect & 1.37 & & & $-0.21(0.25)$ & & \\
\hline \multirow[t]{4}{*}[100]{$\mathrm{Fe}-\mathrm{Ni}$} & $1 \mathrm{nnt}$ & 1.43 & 0.92 & -0.15 & $2.03(2.03)$ & $1.34(1.34)$ & $-0.15(0.15)$ \\
\hline & $1 \mathrm{nncFe}$ & -1.29 & -0.90 & 0.11 & $-0.44(0.76)$ & $-0.59(0.59)$ & $0.18(0.18)$ \\
\hline & $1 \mathrm{nncNi}$ & -1.28 & -0.91 & 0.11 & $-1.17(1.36)$ & $-0.59(0.59)$ & $0.11(0.11)$ \\
\hline & Defect & 1.37 & & & $0.74(0.73)$ & & $-0.07(0.11)$ \\
\hline \multirow[t]{4}{*}{ [010]Fe-Ni } & $1 \mathrm{nnt}$ & -1.19 & -0.95 & 0.12 & $-2.05(2.05)$ & $-1.30(1.30)$ & $0.08(0.08)$ \\
\hline & $1 \mathrm{nncFe}$ & 0.08 & -0.26 & -0.02 & $-0.17(0.85)$ & $0.05(0.38)$ & $-0.13(0.17)$ \\
\hline & $1 \mathrm{nncNi}$ & 0.08 & -0.26 & -0.02 & $-0.16(1.36)$ & $-0.13(0.52)$ & $-0.11(0.17)$ \\
\hline & Defect & 1.51 & & & $-0.65(0.65)$ & & $-0.21(0.21)$ \\
\hline \multirow[t]{4}{*}[100]{$\mathrm{Fe}-\mathrm{Cr}$} & $1 \mathrm{nnt}$ & 1.46 & 0.77 & -0.10 & $1.85(1.85)$ & $0.82(0.83)$ & $-0.08(0.09)$ \\
\hline & $1 \mathrm{nncFe}$ & -1.21 & -0.98 & 0.11 & $-0.50(0.89)$ & $-0.63(0.63)$ & $0.11(0.12)$ \\
\hline & $1 \mathrm{nncCr}$ & -1.20 & -0.99 & 0.12 & $-0.13(0.57)$ & $-0.59(0.59)$ & 0.09 (0.09) \\
\hline & Defect & 1.51 & & & $0.20(0.22)$ & $0.11(0.18)$ & \\
\hline \multirow[t]{4}{*}[010]{$\mathrm{Fe}-\mathrm{Cr}$} & $1 \mathrm{nnt}$ & -1.19 & -0.95 & 0.12 & $-1.88(1.88)$ & $-1.15(1.15)$ & 0.09 (0.09) \\
\hline & $1 \mathrm{nncFe}$ & 0.08 & -0.26 & -0.02 & $0.01(0.98)$ & $-0.06(0.39)$ & $-0.06(0.12)$ \\
\hline & $1 \mathrm{nncCr}$ & 0.08 & -0.26 & -0.02 & $-0.22(0.69)$ & $-0.05(0.34)$ & $-0.03(0.10)$ \\
\hline & Defect & 1.51 & & & $-0.01(0.11)$ & $0.13(0.16)$ & \\
\hline
\end{tabular}


by atoms positioned at defect sites and their $1 \mathrm{nn}$ sites, and in Table VI, which summarizes the same information but for all the point defects studied and compared to bulk calculations. It can be seen in Table VI that whatever the orientation of the dumbbell axis, the magnitude of the moments carried by the $\mathrm{Fe}$ and $\mathrm{Cr}$ atoms has been globally reduced for the atoms in the compressive sites (1nnc) and enhanced for those in the tensile sites (1nnt) by the introduction of the defect. Furthermore, the moments carried by the dumbbell atoms are significantly reduced compared to the moment of the Fe atom which was at the site the dumbbell was created (defect-free reference state). These trends are in accordance with previous studies on bcc Fe. ${ }^{58}$ Finally, the moments of Fe dumbbells are more reduced when introduced along the [010] direction (negative sign on average) rather than along the [100] direction (positive sign on average). To be more specific, as can be easily seen in Fig. 8, most of the Fe-Fe dumbbell atoms introduced along the [100] axis have positive moments $\left(0.37 \mu_{B}\right)$, whereas their first nearest $\mathrm{Cr}$ and $\mathrm{Ni}$ exhibit on average, in compressive sites, negative $\left(-0.61 \mu_{B}\right)$ and positive $\left(0.16 \mu_{B}\right)$ moments, respectively. In this case, the moment interactions between the point defects and these atoms seem to be favorable since, on average, $\mathrm{Fe}$ atoms of dumbbells have antiferromagnetic interactions with $\mathrm{Cr}$ atoms and ferromagnetic interactions with $\mathrm{Ni}$ atoms as in Fig. 2. When Fe-Fe dumbbell atoms are introduced along the [010] axis, the situation is different. $\mathrm{Fe}$ atoms of the dumbbells have an average moment $-0.21 \mu_{B}$ and almost no interaction with $\mathrm{Cr}$ and $\mathrm{Ni}$ atoms (whose moments are, respectively, on average $0.03 \mu_{B}$ and $\left.-0.11 \mu_{B}\right)$. In this case, almost one-third of the Fe atoms in the compressive sites have flipped spins, and the effect of the $\mathrm{Ni}$ and $\mathrm{Cr}$ atoms are thus less important.

It can be noted that the reference state seems to be more stable against the addition of a [010] dumbbell than that of a [100] dumbbell. For the latter, significant instabilities in the moments of the $\mathrm{Fe}$ atoms in compressive sites can be seen in Fig. 7. Indeed, $\mathrm{Fe}$ atoms positioned in compressive sites of [100] oriented dumbbells are prone to important moment variations, which can even sometimes alter locally the AFM1 ordering, as shown previously. This is not observed for the [010] oriented dumbbells. Thus, the AFM1 magnetic structure seems more robust against addition of [010] Fe-Fe dumbbells, which may account for the better CC values obtained systematically for the LRs. This behavior can be easily understood since [010] dumbbell atoms lie inside AFM1 planes, whereas [100] dumbbell atoms lie in between two magnetic planes, thus breaking the AFM1 symmetry. Moments carried by [100] dumbbell atoms are therefore expected to be more prone to unpredictable variations owing to their proximity with both magnetic planes "up" and "down." This behavior has been confirmed by performing dumbbell calculations with different initial magnetic configurations (up-up, up-down, and down-down) which led to different final moments for the dumbbell atoms.

Despite the different magnetic interactions found between [100] and [010] oriented dumbbells and knowing the key role played by atoms in compressive sites in the evaluation of the formation energy as shown by the LRs presented previously, we find that the dumbbell formation energies are nevertheless similar on average whether the dumbbell is oriented along the [100] or the [010] direction, as can be seen in Table III. This result suggests that it is the chemical composition of the first shell of atoms surrounding the Fe-Fe dumbbells which provides the largest contribution to the formation energy values and that the magnetic interactions between dumbbell atoms and their $1 \mathrm{nn}$ in tensile or compressive sites constitute secondorder effects.

\section{Mixed dumbbell formation energies}

In this section, the results obtained for mixed dumbbells are analyzed. As two different atoms form the dumbbells, the position of the atoms in compressive sites relative to the mixed dumbbells was also considered in the LR variables.

The parameter values presented for $\Delta E_{\mathrm{LR}}^{\mathrm{Fe}-\mathrm{Ni}}$ and $\Delta E_{\mathrm{LR}}^{\mathrm{Fe}-\mathrm{Cr}}$ in Table $\mathrm{V}$ follow the same trends as those observed for the $\mathrm{Fe}-\mathrm{Fe}$ dumbbells. First of all, $\mathrm{Cr}$ and $\mathrm{Ni}$ positioned in compressive sites were also found to impact strongly on the calculated formation energies. Furthermore, in this case, the more one differentiates between the different types of sites that are $1 \mathrm{nn}$ to the mixed dumbbells, the better the estimates are of the point defect formation energies. Thus, the best CCs were obtained by differentiating between the compressive sites located closer to the $\mathrm{Fe}$ or closer to the solute atom of the dumbbell, i.e., by considering both $1 \mathrm{nncFe} \_A$ and $1 \mathrm{nncS} \_A$ variables, respectively, instead of the $1 \mathrm{nnc} \_A$ variable defined in Table IV. The analysis of the parameter values assigned to these variables shows that the formation energies depend on how the $\mathrm{Cr}$ and $\mathrm{Ni}$ atoms in compression sites are positioned with respect to the mixed dumbbell. Indeed, parameters presented in Table $\mathrm{V}$ for $\Delta E_{\mathrm{LR}}^{\mathrm{Fe}-\mathrm{Ni}}$ and $\Delta E_{\mathrm{LR}}^{\mathrm{Fe}-\mathrm{Cr}}$ can have similar as well as different values depending on the location of the $1 \mathrm{nn}$ in compressive sites relative to the dumbbell atoms.

In the case of the Fe-Ni [100] dumbbell, for instance, the presence of $\mathrm{Cr}$ atoms positioned in compressive sites tends to decrease the formation energy whatever their positions are relative to the mixed dumbbell. This tendency seems to be more pronounced when $\mathrm{Cr}$ atoms are located closer to the $\mathrm{Fe}$ atom in the dumbbell (the magnitudes of the parameter values assigned to the 1 nncFe_Cr variable are larger), but as in the case of $\langle 100\rangle \mathrm{Fe}-\mathrm{Fe}$ dumbbells, slight variations in the LR parameters render very similar CCs, thus making the results difficult to interpret. Parameter values assigned to the number of $\mathrm{Ni}$ atoms in compressive sites, however, have opposite signs depending on their location. The occurrence of $\mathrm{Ni}$ atoms in compressive sites located next to the Fe atom of the [100] Fe-Ni dumbbell tends to increase the formation energy, as was found for the $\langle 100\rangle \mathrm{Fe}-\mathrm{Fe}$ dumbbell in the previous section. However, when located next to the $\mathrm{Ni}$ atom of the [100] Fe-Ni dumbbell, they tend to decrease the formation energy. These more energetically favorable interactions found for Ni-Ni pairs are in agreement with results obtained by Klaver et al., ${ }^{23}$ which generally found a modest attraction of $\mathrm{Ni}-\mathrm{Ni}$ pairs in pure fcc Fe. No such behavior was observed for the [010] Fe-Ni dumbbell, for which both $\mathrm{Cr}$ and $\mathrm{Ni}$ atoms in compressive sites seem to have similar influence on the formation energy, whatever their positions relative to the mixed dumbbells. These different effective interactions found between the $\mathrm{Ni}$ atom in the dumbbell (oriented either along the [100] or the [010] axis) and its first nearest $\mathrm{Ni}$ in compressive sites, though, do not 
necessarily contradict each other owing to the strong influence of the magnetic state on the binding energies found by Klaver et $a l .{ }^{23}$ In particular, $\mathrm{Ni}-\mathrm{Ni}$ pairs generally exhibited modest attractions in the AFM1 state but repulsive binding in the AFMD state.

In the case of the Fe-Cr dumbbells, we found that it is necessary to take into account the position of $\mathrm{Cr}$ and $\mathrm{Ni}$ atoms in compression sites relative to the mixed dumbbell to obtain good estimates of the DFT formation energies. Indeed, whatever the direction of the dumbbell axis, bad estimates (small $\mathrm{CC}$ values) were found otherwise. The $\mathrm{CC}$ values are found to be considerably enhanced by describing more accurately the first shell surrounding the Fe-Cr dumbbells. Indeed, the addition of one $\mathrm{Cr}$ in the dumbbell seems, whatever the dumbbell orientation, to have a strong impact on the behavior of the local environment. In particular, the parameter values of the two variables describing the effective interactions between $\mathrm{Cr}$ atoms in compressive sites and the two atoms of the dumbbells (1nncS_Cr and $1 \mathrm{nncFe} C \mathrm{Cr}$ variables) exhibit opposite signs. Negative values are obtained when the $\mathrm{Cr}$ atoms in compressive sites are close to the Fe atom of the dumbbell which indicates favorable interactions. However, when the $\mathrm{Cr}$ atoms in compressive sites are close to the $\mathrm{Cr}$ atom of the mixed dumbbell, these interactions are unfavorable, in agreement with the strong $\mathrm{Cr}-\mathrm{Cr}$ repulsion in pure fcc $\mathrm{Fe}$ for three different magnetic structures. ${ }^{23}$ It may be mentioned that very similar repulsive interactions are also found for $\mathrm{Cr}-\mathrm{Cr}$ pairs in bcc FM Fe (Refs. 58 and 59) at short separation distances.

It is worth mentioning that magnetic moments of the Fe, $\mathrm{Cr}$, and $\mathrm{Ni}$ atoms $1 \mathrm{nn}$ to the mixed dumbbell follow the same trends as those displayed for the $\langle 100\rangle \mathrm{Fe}-\mathrm{Fe}$ dumbbell, as can be seen in Table VI. Indeed, moments measured in the reference state are, on average, enhanced with the addition of the [100] oriented dumbbell, whereas with the addition of the [010] oriented dumbbell, the moment amplitudes are generally reduced in both compressive and tensile sites. The solute atom in mixed dumbbells can have a strong impact on the moments measured on atoms in the compressive sites as can be easily seen in Fig. 9. In particular, the Ni atom of Fe-Ni dumbbells tends to decrease the values of Fe moments in the compressive sites. The moments also depend on the location of the $1 \mathrm{nn}$ in compressive sites relative to the dumbbell atoms. This feature is particularly significant for the mixed dumbbells oriented along the [100] axis for which Fe moments are weaker when located next to the $\mathrm{Ni}$ atom of the Fe-Ni dumbbell and greater

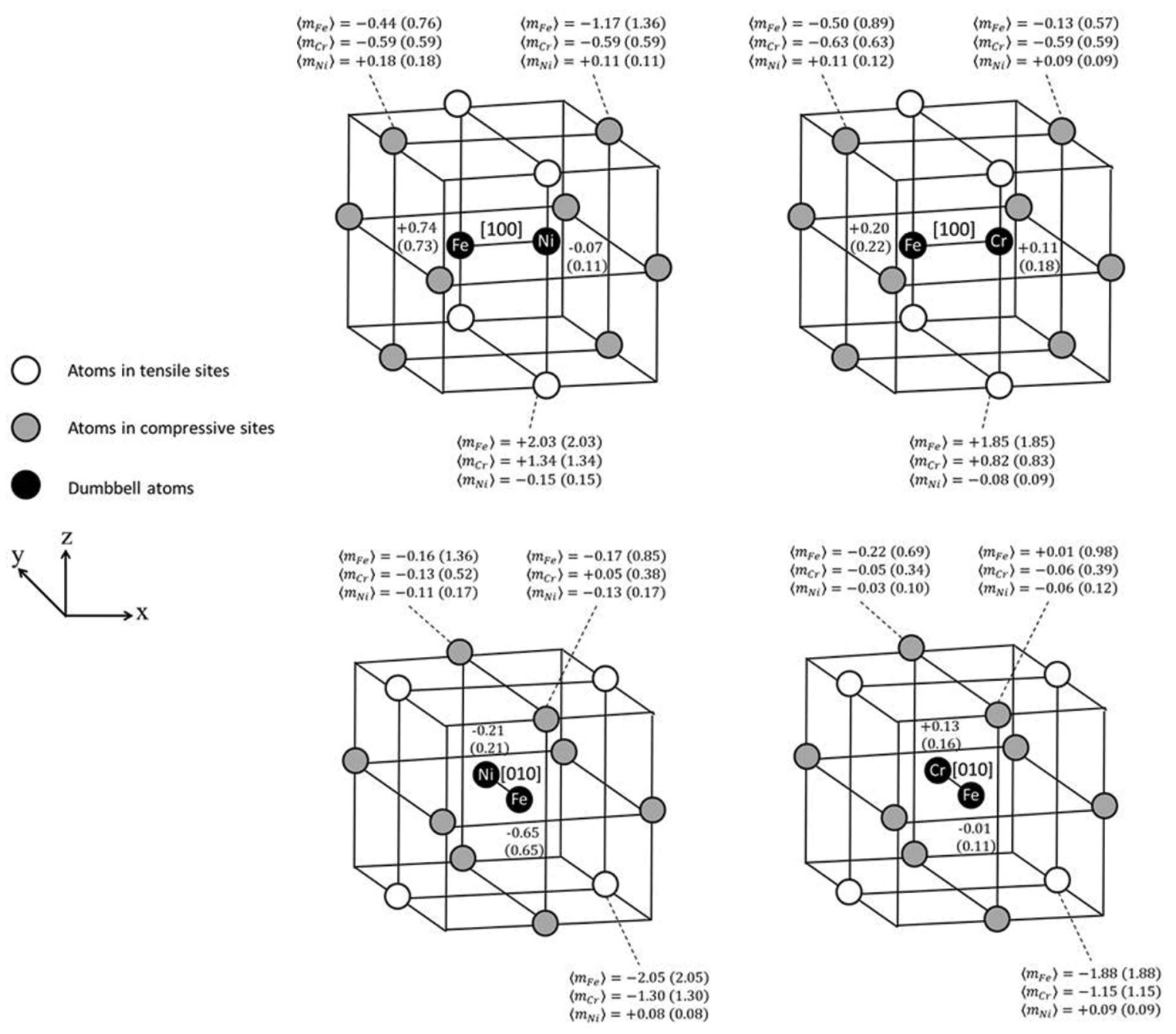

FIG. 9. Average values of DFT magnetic moments (in $\mu_{B}$ ), $\langle m\rangle$, for atoms that are $1 \mathrm{nn}$ to $\langle 100\rangle$ mixed dumbbells. The values displayed in parentheses refer to the mean value of the absolute of moments. 
when located next to the $\mathrm{Cr}$ atom of the $\mathrm{Fe}-\mathrm{Cr}$ dumbbell, relative to the case of [100] $\mathrm{Fe}-\mathrm{Fe}$ dumbbells. Furthermore, the mean values of moments found for the Fe atom of the mixed dumbbell are on average, respectively, positive for the [100] oriented dumbbell and negative for the [010] dumbbell, as previously found for the $\langle 100\rangle \mathrm{Fe}-\mathrm{Fe}$ dumbbells. Their mean amplitudes are, however, greater for the Fe-Ni dumbbells and weaker for the $\mathrm{Fe}-\mathrm{Cr}$ dumbbells relative to those measured in the Fe-Fe dumbbells. The mean values of moments carried by the $\mathrm{Ni}$ atom of the mixed dumbbell are negative for both kinds of dumbbell. Lower values are nevertheless found for the $\mathrm{Ni}$ atom of the [010] oriented dumbbell. These results underline again the significant impact of the direction of the dumbbell axis. By contrast, the mean values of moments carried by the $\mathrm{Cr}$ atom of the mixed dumbbell are positive for both kinds of dumbbell and are of equal magnitude.

\section{CONCLUSIONS}

We have determined point defect formation energies in an fcc Fe-10Ni-20Cr model alloy. In order to select the most appropriate reference state, we modeled PM, AFM1, AFMD, and FM structures. The most stable state obtained using DFT is AFM1, which was therefore used for the point defect calculations. A study of this reference state showed that the moments of the $\mathrm{Cr}$ and $\mathrm{Ni}$ atoms are ruled by the moments of their nearest-neighbor Fe atoms. We estimated $\mu_{A}^{\mathrm{Fe}-10 \mathrm{Ni}-20 \mathrm{Cr}}(A$ refers to the element type) chemical potentials for $\mathrm{Fe}, \mathrm{Ni}$, and $\mathrm{Cr}$ atoms, by calculating the minimum of substitution energy on most of the $180 \mathrm{Fe}, 50 \mathrm{Cr}$, and $26 \mathrm{Ni}$ atoms sites. Our extensive set of point defect calculations based on DFT shows a large energy range for each set of point defect formations, thus underlying the importance of the local environment in concentrated alloys. In particular, we underline the key role played by the atoms of the first shell surrounding the point defects in the formation energy values. The main results can be summarized as follows:

(1) The simple knowledge of the number of $\mathrm{Ni}$ and $\mathrm{Cr}$ atoms $1 \mathrm{nn}$ to the vacancy ( $1 \mathrm{nn} \_\mathrm{Ni}$ and $1 \mathrm{nn} \_\mathrm{Cr}$, respectively) is sufficient to estimate with a reasonable agreement the DFT data. The vacancy formation energy decreases (increases) with increasing amount of $\mathrm{Ni}(\mathrm{Cr})$ neighbors.

(2) Inversely, linear regressions usually showed that the more first neighbor $\mathrm{Ni}$ atoms the larger the dumbbell formation energy, whereas the more first neighbor $\mathrm{Cr}$ atoms the lower the dumbbell formation energy.

(3) Dumbbell formation energies are best estimated by linear regressions using the variables describing the effective interactions between $\mathrm{Cr}$ and $\mathrm{Ni}$ atoms in compressive sites and for the dumbbell atoms (i.e., 1nnc_Ni and $1 \mathrm{nnc}$-Cr for self-interstitials and $1 \mathrm{nncFe} \_\mathrm{Ni}, 1 \mathrm{nncFe} \_\mathrm{Cr}, 1 \mathrm{nncS} \_\mathrm{Ni}$, and 1nncS_Cr for mixed dumbbells).

(4) The chemical composition of the first shell of atoms surrounding the Fe-Fe dumbbells provides the largest contribution to the formation energy values, whereas magnetic interactions between dumbbell atoms and their $1 \mathrm{nn}$ in tensile or compressive sites seem to present second-order effects.

(5) The interactions of $\mathrm{Ni}$ and $\mathrm{Cr}$ solutes with point defects in fcc $\mathrm{Fe}-10 \mathrm{Ni}-20 \mathrm{Cr}$ are consistent with what would intuitively be expected of moderately oversized and undersized solutes, respectively. $\mathrm{Ni}$ binds to the vacancy and is generally repelled from the compressive sites of $\langle 100\rangle \mathrm{Fe}-\mathrm{Fe}$ dumbbells but shows positive binding in the tensile sites. $\mathrm{Cr}$ exhibits the opposite tendencies. The same conclusions can be drawn for the selfinterstitial atom formation energies. Indeed, $\mathrm{Ni}$ is very unlikely to be found in mixed Fe-Ni dumbbells or in Ni-Ni dumbbells. $\mathrm{Cr}$, on the other hand, can form mixed dumbbells. These results are not, however, in agreement with the experimentally derived size factors for $\mathrm{Ni}$ and $\mathrm{Cr}$ solutes in type $316 \mathrm{SS}$ and in the pure materials. $^{57}$

(6) A few differences can be reported for the mixed dumbbells compared to $\langle 100\rangle \mathrm{Fe}-\mathrm{Fe}$ dumbbells. In particular, when located next to the $\mathrm{Ni}$ atom of the [100] Fe-Ni dumbbell, $\mathrm{Ni}$ atoms tend to decrease the formation energy. Similarly, located next to the $\mathrm{Cr}$ atom of the [100] $\mathrm{Fe}-\mathrm{Cr}$ dumbbell, $\mathrm{Cr}$ atoms tend to increase the formation energy. These results are consistent with the strong $\mathrm{Cr}-\mathrm{Cr}$ repulsion and the energetically favorable interactions found for $\mathrm{Ni}-\mathrm{Ni}$ pairs by Klaver et al. in pure fcc Fe. ${ }^{23}$

These results are of interest as they provide insight about interactions which occur in fcc Fe-Ni-Cr model concentrated alloys. In particular, such information is required to build reliable cohesive models to model the microstructure evolution of concentrated austenitic stainless steels used in the nuclear industry.

\section{ACKNOWLEDGMENTS}

This work was sponsored by the EU-FP7 PERFORM-60 project (Grant No. 232612) and by EPSRC through the UKCP Collaboration.

\section{APPENDIX: CALCULATION OF CHEMICAL POTENTIALS}

Although naturally defined at constant temperature and pressure, the chemical potential can also be calculated in constant volume and temperature simulations:

$$
\left.\frac{\partial F}{\partial N_{A}}\right|_{T, V, N_{B \neq A}}=\mu_{A} \text {. }
$$

At finite temperature, the excess chemical potential differences $\Delta \mu_{\mathrm{ex}}=\left(\mu_{B}-\mu_{B}^{\mathrm{id}}\right)-\left(\mu_{A}-\mu_{A}^{\mathrm{id}}\right)$, where $\mu_{B}$ refers to the chemical potential of species $B$ and $\mu_{B}^{\mathrm{id}}$ refers to the ideal contribution of $\mu_{B}$, are usually calculated using Widom-type substitution techniques ${ }^{47}$ which, in principle, can be done either at constant pressure or at constant volume. This consists of simulating the nonsubstituted reference system " $A$ " of energy $U_{A}$ (containing $N_{A}$ atoms of type $A$ and $N_{B}$ atoms of type $B$ ) with a Monte Carlo procedure, and estimating the excess chemical potential difference by canonical averages of the exponential of the substitution energy $\Delta^{A \rightarrow B} E=$ $E_{B}-E_{A}$ :

$$
\Delta^{A \rightarrow B} \mu_{\mathrm{ex}}=-\beta^{-1} \ln \left\langle\exp \left(-\beta \Delta^{A \rightarrow B} E\right)\right\rangle_{A},
$$

where $\beta=\left(k_{B} T\right)^{-1}$ and \langle\rangle$_{A}$ denotes the canonical average on the " $A$ " system configurations. Stating the ratio of the ideal gas contributions gives ${ }^{60}$

$$
\Delta^{A \rightarrow B} \mu=-\beta^{-1} \ln \left\langle\frac{N_{B}}{N_{A}+1} \exp \left(-\beta \Delta^{A \rightarrow B} E\right)\right\rangle_{A} .
$$


In practice, to obtain accurate estimates of the chemical potential differences, it is safer to realize the inverse substitution starting from a " $B$ " system of energy $E_{B}$ with an additional $B$ atom (containing $N_{A}-1 A$-type atoms and $N_{B}+1 B$-type atoms) as a reference, and to make a canonical average on the " $B$ to $A$ " substitution energies $\Delta^{B \rightarrow A} E=E_{A}-E_{B}$ :

$$
\Delta^{B \rightarrow A} \mu=-\beta^{-1} \ln \left\langle\frac{N_{A}}{N_{B}+1} \exp \left(-\beta \Delta^{B \rightarrow A} E\right)\right\rangle_{B} .
$$

For the purpose of calculating defect formation energies from Eq. (1) to Eq. (3), calculations of the chemical potentials of the species involved in the defects are required. Precise calculations of chemical potentials by DFT methods would be extremely time consuming. This would require, depending on temperature, sampling a large number of configurations by a Monte Carlo Metropolis procedure. Thus, the estimates of chemical potentials we present are in fact only zero Kelvin reference energies in VASP for the addition or the removal of a given type of atom in our AFM1 reference state (simply called chemical potential and referred to as $\mu_{A}^{\mathrm{Fe}-10 \mathrm{Ni}-20 \mathrm{Cr}}$, where $A$ refers to the element type), thus neglecting any entropic contribution. The zero Kelvin equivalent of Widom-type substitution techniques and associated Monte Carlo procedure for estimates of $\Delta \mu$ would be to estimate it from the minimum value of substitution energies sampled. Indeed, the limit of Eq. (A1) for $\beta^{-1} \underset{T \rightarrow 0}{\rightarrow} 0$, the ideal gas contribution vanishes and

$$
\begin{aligned}
& \Delta^{A \rightarrow B} \mu=-\beta^{-1} \ln \left\langle\exp \left(-\beta \Delta^{A \rightarrow B} E\right)\right\rangle_{A} \\
& \approx-\beta^{-1} \ln \left[\frac{1}{n} \sum_{i=1}^{n} \exp \left(-\beta \Delta^{A \rightarrow B} E_{i}\right)\right] \\
&=-\beta^{-1} \ln \left(\frac{1}{n} \exp \left[-\beta \min _{k}\left(\Delta^{A \rightarrow B} E_{k}\right)\right] \sum_{i=1}^{n} \exp \left\{-\beta\left[\Delta^{A \rightarrow B} E_{i}-\min _{k}\left(\Delta^{A \rightarrow B} E_{k}\right)\right]\right\}\right) \\
&=-\beta^{-1} \ln \left[\frac{1}{n} \exp \left[-\beta \min _{k}\left(\Delta^{A \rightarrow B} E_{k}\right)\right]\left(1+\sum_{i=1, i \neq j}^{n} \exp \left\{-\beta\left[\Delta^{A \rightarrow B} E_{i}-\min _{k}\left(\Delta^{A \rightarrow B} E_{k}\right)\right]\right\}\right)\right] \\
&=-\beta^{-1} \ln \left\{\exp \left[-\beta \min _{k}\left(\Delta^{A \rightarrow B} E_{k}\right)\right]\right\}-\beta^{-1} \ln \left(\frac{1}{n}\right)-\beta^{-1} \ln \left[1+\sum_{i=1, i \neq j}^{n} \exp \left(-\beta \varepsilon_{i}\right)\right] \\
& \underset{\beta^{-1} \rightarrow 0}{\rightarrow} \min _{k}\left(\Delta^{A \rightarrow B} E_{k}\right),
\end{aligned}
$$

where $j$ is such that $\Delta^{A \rightarrow B} E_{i}=\min _{k}\left(\Delta^{A \rightarrow B} E_{k}\right), \varepsilon_{i}=\Delta^{A \rightarrow B} E_{i}-\min _{k}\left(\Delta^{A \rightarrow B} E_{k}\right)>0$ for any $i \neq j$, and where the sum over $k$ is made on the substitution energies sampled in the AFM1 configuration. Similarly, we have $\Delta^{B \rightarrow A} \mu \underset{\beta^{-1} \rightarrow 0}{\rightarrow} \min _{k}\left(\Delta^{B \rightarrow A} E_{k}\right)$.

${ }^{1}$ R. A. Lula, J. G. Parr, and A. Hanson, Stainless Steel (American Society for Metals, Metals Park, OH, 1986).

${ }^{2}$ O. Dimitrov and C. Dimitrov, J. Nucl. Mater. 105, 39 (1982).

${ }^{3}$ C. Dimitrov and O. Dimitrov, J. Phys. F: Met. Phys. 14, 793 (1984).

${ }^{4}$ C. Dimitrov, A. Benkaddour, O. Dimitrov, C. Corbel, and P. Moser, Mater. Sci. Forum 15-18, 1275 (1987).

${ }^{5}$ C. Dimitrov and O. Dimitrov, J. Nucl. Mater. 152, 21 (1988).

${ }^{6}$ A. Benkaddour, C. Dimitrov, and O. Dimitrov, J. Nucl. Mater. 217, 118 (1994).

${ }^{7}$ C. Dimitrov, M. Tenti, and O. Dimitrov, J. Phys. F: Met. Phys. 11, 753 (1981).

${ }^{8}$ H. L. Meyerheim, R. Popescu, D. Sander, and J. Kirschner, Phys. Rev. B 71, 035409 (2005).

${ }^{9}$ Y. Tsunoda, H. Nogami, and M. Takasaka, Phys. Rev. B 76, 054419 (2007).

${ }^{10}$ W. A. Hines, P. Shanthakumar, T. Huang, J. I. Budnick, R. L. Miller, D. M. Pease, and D. M. Perry, Phys. Status Solidi B 246, 2154 (2009).

${ }^{11}$ S. C. Abrahams, L. Guttman, and J. S. Kasper, Phys. Rev. 127, 2052 (1962).
${ }^{12}$ Y. Tsunoda, J. Phys.: Condens. Matter 1, 10427 (1989).

${ }^{13}$ H. C. Herper, E. Hoffmann, and P. Entel, Phys. Rev. B 60, 3839 (1999).

${ }^{14}$ D. Spišák and J. Hafner, Phys. Rev. B 61, 16129 (2000); Phys. Rev. Lett. 88, 056101 (2002).

${ }^{15}$ C. Domain and C. S. Becquart, Phys. Rev. B 65, 024103 (2002).

${ }^{16}$ D. E. Jiang and E. A. Carter, Phys. Rev. B 67, 214103 (2003).

${ }^{17}$ L. T. Kong and B. X. Liu, J. Alloys Compd. 414, 36 (2006).

${ }^{18}$ V. P. Antropov, M. I. Katsnelson, M. van Schilfgaarde, and B. N. Harmon, Phys. Rev. Lett. 75, 729 (1995).

${ }^{19}$ K. Knöpfle, L. M. Sandratskii, and J. Kübler, Phys. Rev. B 62, 5564 (2000).

${ }^{20}$ E. Sjöstedt and L. Nordström, Phys. Rev. B 66, 014447 (2002).

${ }^{21}$ A. K. Majumdar and P. V. Blanckenhagen, Phys. Rev. B 29, 4079 (1989).

${ }^{22}$ F. Körmann, A. Dick, B. Grabowski, T. Hickel, and J. Neugebauer, Phys. Rev. B 85, 125104 (2012).

${ }^{23}$ T. P. C. Klaver, D. J. Hepburn, and G. J. Ackland, Phys. Rev. B 85, 174111 (2012)

${ }^{24}$ R. Hafner, D. Spisák, R. Lorenz, and J. Hafner, J. Phys.: Condens. Matter 13, L239 (2001). 
${ }^{25}$ S. Cottenier, B. De Vries, J. Meersschaut, and M. Rots, J. Phys.: Condens. Matter 14, 3275 (2002).

${ }^{26}$ R. Hafner, D. Spisák, R. Lorenz, and J. Hafner, Phys. Rev. B 65, 184432 (2002).

${ }^{27}$ L. Vitos, P. A. Korzhavyi, and B. Johansson, Phys. Rev. Lett. 88, 155501 (2002).

${ }^{28}$ L. Vitos, P. A. Korzhavyi, and B. Johansson, Nat. Mater. 2, 25 (2003).

${ }^{29}$ L. Vitos, P. A. Korzhavyi, and B. Johansson, Phys. Rev. Lett. 96, 117210 (2006).

${ }^{30}$ L. Delczeg, B. Johansson, and L. Vitos, Phys. Rev. B 85, 174101 (2012).

${ }^{31}$ H. Pitkanen, M. Alatalo, A. Puisto, M. Ropo, K. Kokko, M. P. J. Punkkinen, P. Olsson, B. Johansson, S. Hertzman, and L. Vitos, Phys. Rev. B 79, 024108 (2009).

${ }^{32}$ A. Zunger, S.-H. Wei, L. G. Ferreira, and J. E. Bernard, Phys. Rev. Lett. 65, 353 (1990).

${ }^{33}$ S. M. Foiles, M. I. Baskes, and M. S. Daw, Phys. Rev. B 33, 7983 (1986).

${ }^{34}$ L. Malerba, G. J. Ackland, C. S. Becquart, G. Bonny, C. Domain, S. L. Dudarev, C.-C. Fu, D. Hepburn, M. C. Marinica, P. Olsson, R. C. Pasianot, J. M. Raulot, F. Soisson, D. Terentyev, E. Vincent, and F. Willaime, J. Nucl. Mater. 406, 7 (2010).

${ }^{35}$ G. J. Ackland, G. I. Tichy, V. Vitek, and M. W. Finnis, Philos. Mag. A 56, 735 (1987).

${ }^{36}$ A. De Vita and M. J. Gillan, J. Phys.: Condens. Matter 3, 6225 (1991).

${ }^{37}$ J. D. Tucker, R. Najafabadi, T. R. Allen, and D. Morgan, J. Nucl. Mater. 405, 216 (2010).

${ }^{38}$ G. Kresse and J. Hafner, Phys. Rev. B 47, 558 (1993).

${ }^{39}$ G. Kresse and J. Furthmüller, Phys. Rev. B 54, 11169 (1996).

${ }^{40}$ P. E. Blöchl, Phys. Rev. B 50, 17953 (1994).

${ }^{41}$ G. Kresse and D. Joubert, Phys. Rev. B 59, 1758 (1999).
${ }^{42}$ J. P. Perdew and A. Zunger, Phys. Rev. B 23, 5048 (1981).

${ }^{43}$ B. Liu, in Numerical Algorithms in Chemistry: Algebraic Methods, edited by C. Moler and I. Shavitt (Lawrence Berkeley Laboratory, University of California, 1978), p. 49; E. R. Davidson, in Methods in Computational Molecular Physics, edited by G. H. F. Diercksen and S. Wilson, NATO Advanced Study Institute, Series C, Vol. 113 (Plenum, New York, 1983), p. 95.

${ }^{44}$ B. Alling, T. Marten, and I. A. Abrikosov, Phys. Rev. B 82, 184430 (2010).

${ }^{45}$ P. Steneteg, B. Alling, and I. A. Abrikosov, Phys. Rev. B 85, 144404 (2012).

${ }^{46}$ M. Methfessel and A. T. Paxton, Phys. Rev. B 40, 3616 (1989).

${ }^{47}$ B. Widom, J. Chem. Phys. 39, 2808 (1963).

${ }^{48} \mathrm{G}$. Adjanor, M. Athènes, and J. M. Rodgers, J. Chem. Phys. 135, 044127 (2011)

${ }^{49}$ C. H. Bennett, J. Comput. Phys. 22, 245 (1976).

${ }^{50}$ T. P. C. Klaver, R. Drautz, and M. W. Finnis, Phys. Rev. B 74, 094435 (2006).

${ }^{51}$ P. Olsson, T. P. C. Klaver, and C. Domain, Phys. Rev. B 81, 054102 (2010).

${ }^{52}$ P. James, O. Eriksson, B. Johansson, and I. A. Abrikosov, Phys. Rev. B 59, 419 (1999).

${ }^{53}$ M. Ekholm and I. A. Abrikosov, Phys. Rev. B 84, 104423 (2011).

${ }^{54}$ A. Marucco, Mater. Sci. Eng., A 189, 267 (1994).

${ }^{55}$ M. Hayase, M. Shiga, and Y. Nakamura, J. Phys. Soc. Jpn. 34, 925 (1973).

${ }^{56}$ See http://steelfinder.outokumpu.com/

${ }^{57}$ J. L. Straalsund and J. F. Bates, Metall. Trans. 5, 493 (1974).

${ }^{58}$ P. Olsson, C. Domain, and J. Wallenius, Phys. Rev. B 75, 014110 (2007).

${ }^{59}$ T. P. C. Klaver, P. Olsson, and M. W. Finnis, Phys. Rev. B 76, 214110 (2007).

${ }^{60}$ D. Frenkel and B. Smit, Understanding Molecular Simulation (Academic Press, New York, 1996). 